University of Wollongong

Research Online

Faculty of Business - Papers (Archive)

Faculty of Business and Law

$1-1-2016$

Endogenous growth and technological progress with innovation driven by social interactions

Simone Marsiglio

University of Wollongong, simonem@uow.edu.au

Marco Tolotti

Ca'Foscari University of Venice, tolotti@unive.it

Follow this and additional works at: https://ro.uow.edu.au/buspapers

Part of the Business Commons

Research Online is the open access institutional repository for the University of Wollongong. For further information contact the UOW Library: research-pubs@uow.edu.au 


\title{
Endogenous growth and technological progress with innovation driven by social interactions
}

\begin{abstract}
We analyze the implications of innovation and social interactions on economic growth in a stylized endogenous growth model with heterogeneous research firms. A large number of research firms decide whether to innovate or not, by taking into account what competitors (i.e., other firms) do. This is due to the fact that their profits partly depend on an externality related to the share of firms which actively engage in research activities. Such a share of innovative firms also determines the evolution of technology in the macroeconomy, which ultimately drives economic growth. We show that when the externality effect is strong enough multiple BGP equilibria may exist. In such a framework, the economy may face a low growth trap suggesting that it may end up in a situation of slow long-run growth; however, such an outcome may be fully solved by government intervention. We also show that whenever multiple BGP exist, they are metastable meaning that the economy may cyclically fluctuate between the low and high BGP as a result of shocks affecting the individual behavior of research firms.

\section{Disciplines \\ Business}

\section{Publication Details}

Marsiglio, S. \& Tolotti, M. (2018). Endogenous growth and technological progress with innovation driven by social interactions. Economic Theory, 65 (2), 293-328.
\end{abstract}




\title{
Endogenous Growth and Technological Progress with Innovation Driven by Social Interactions*
}

\author{
Simone Marsiglio ${ }^{\dagger} \quad$ Marco Tolotti ${ }^{\ddagger}$ \\ Forthcoming in Economic Theory
}

\begin{abstract}
We analyze the implications of innovation and social interactions on economic growth in a stylized endogenous growth model with heterogenous research firms. A large number of research firms decide whether to innovate or not, by taking into account what competitors (i.e., other firms) do. This is due to the fact that their profits partly depend on an externality related to the share of firms which actively engage in research activities. Such a share of innovative firms also determines the evolution of technology in the macroeconomy, which ultimately drives economic growth. We show that when the externality effect is strong enough multiple BGP equilibria may exist. In such a framework, the economy may face a low growth trap suggesting that it may end up in a situation of slow long run growth; however, such an outcome may be fully solved by government intervention. We also show that whenever multiple BGP exist, they are metastable meaning that the economy may cyclically fluctuate between the low and high BGP as a result of shocks affecting the individual behavior of research firms.
\end{abstract}

Keywords: Economic Growth; Endogenous Fluctuations; Firms Interaction; Innovation; Low Growth Trap; Metastable equilibria

JEL Classification: C60, D70, E32, O40

\section{Introduction}

Technological progress is by far the most important determinant of economic growth in industrialized economies. During the last two decades, after the seminal works of Romer (1986), Aghion \& Howitt (1992), and Grossman \& Helpman (1994), many efforts have been put forward to try understanding and explaining the sources of technological advances. All the resulting works take the nature of the research sector as given, and the interaction among firms in the research industry has never been analyzed in depth thus far 1 . This is however one of the main fields of interest of computational and evolutionary economics; heterogeneous agent models, for instance, may help in explaining how innovation occurs, which are the dynamics of innovation and how innovation determines technological progress (see Dawid (2006) for an extensive survey). The goal of this paper is to bridge these two different branches of literature by developing a stylized but analytically

${ }^{*}$ We are indebted to two anonymous referees for their constructive comments helping us to substantially improve our paper. We also acknowledge the financial support from MIUR under grant "Robust decision making in markets and organizations" (PRIN20103S5RN3) and the support from Ca' Foscari University Venice under the grant "Interactions in complex economic systems: contagion, innovation and crises".

${ }^{\dagger}$ University of Wollongong, School of Accounting, Economics and Finance, Northfields Avenue, Wollongong 2522 NSW, Australia. Contact: simonem@uow.edu.au

${ }^{\ddagger}$ Universitá Ca’ Foscari Venezia, Department of Management, Cannaregio 873, 30121 Venice, Italy. Contact: tolotti@unive.it

${ }^{1}$ Schumpeterian growth models to some extent model the interaction in the research sector by allowing for a business-stealing effect, determining the likelihood that an incumbent innovator loses its monopoly power because of a success in the innovation process by a new entrant (Acemoglu (2009)). Apart from this type of characterization, the endogenous growth literature has not emphasized how the choice of research firms are related and interdependent. 
tractable and micro-founded agent based model of innovation to shed some light on the role that interactions among research firms might play in the process of economic growth. Once an almost traditional economic growth model is extended to allow some form of interaction among research firms along the lines outlined in Brock \& Durlauf (2001) and Blume \& Durlauf (2003), some traditional results, like the uniqueness of equilibrium, found in growth theory vanish. Indeed, such an interaction among research firms, by determining the rate of technological progress, plays a critical role in shaping the whole macroeconomic dynamics. We show that, under certain parameter conditions, the economy may be characterized by a multiplicity of balanced growth path (BGP) equilibria, and a situation of low growth trap. We also show that the economy may eventually (endogenously) fluctuate between the low and high BGP generating thus a growth cycle in which periods of low and high economic growth rates follow one another. Such a cycling behavior is due to the probabilistic nature characterizing the research industry; indeed, under a certain model parametrization the BGP equilibria turn out to be metastable: on a short time scale they appear to be stable attractors, while on a longer time scale unpredictable random jumps lead the economy to sudden shifts towards the other BPG equilibrium. In this context economic policy, aiming to modify the incentives associated with research activities, may be very effective in order to completely solve the low growth trap problem, avoiding also fluctuations in economic activity.

Our paper is thus related to different branches of the economic literature, namely computational and evolutionary economics, economic growth and business cycles theory. From the computational and evolutionary economics literature we simply borrow the interest in analyzing the interaction between research firms and its eventual implications for technological progress and the long run economic growth (Nelson and Winter (1982), Dawid (2006), Dosi et al. (2010)). However, from a methodological point of view our approach is substantially different since we develop a very simple and tractable model, in which most of the results are analytically derived; simulations in our paper play only a marginal role and are instrumental to exemplify some interesting and potential outcomes. Economic growth theory is the main benchmark for our analysis since the model is an almost standard continuous time model of optimal growth with endogenous technological progress $($ Acemoglu $(2009))$. With respect to what traditionally assumed in this literature (Romer (1986), Grossman \& Helpman (1994)), we allow for a certain degree of diffusion in the pattern of innovation, meaning that in our framework technical progress is driven by the interaction among research firms $2^{2}$ To the best of our knowledge, no other study has thus far focused on the firms interaction in the research industry in a way comparable to ours; moreover, all the works identify a unique BGP equilibrium thus cyclical behavior cannot occur ${ }^{3}$. The understanding and characterization of cyclical patterns is the main interest of the business cycle theory ${ }^{4}$ (Kydland and Prescott $(1982)$, King et al. (1988a), King et al. (1988b)), which besides adopting a discrete time framework5 (Evans et al. (1998); Canton (2002); Furukawa

\footnotetext{
${ }^{2}$ This is in line with what suggested by the seminal work by Bass (1969) in the context of diffusion of durables. The Bass model is a particular case of a larger class of epidemiological models. We refer the reader to Hethcote (2000) for a recent survey on the topic.

${ }^{3}$ Few exceptions in which endogenous growth and cyclical fluctuations may be simultaneously experienced exist. Most of these papers focus on an expanding variety model characterized by innovation cycles in which the mechanism underlying economic fluctuations varies from the existence of different investment regimes (Matsuyama (1999) and Matsuyama (2001)) to international trade and foreign spillovers (Furukawa $(2015)$ ). Others focus instead on the mutual relation between human capital investments and productivity growth (Kaas and Zink (2007)). Our approach is substantially different since we rely on a simple capital accumulation model in which the evolution of the total factor productivity is the result of firms' interactions within the research industry.

${ }^{4}$ Cyclical outcomes are also analyzed in growth theory by characterizing the eventual existence of equilibrium indeterminacy (Benhabib and Farmer (1994); Benhabib and Farmer (1998); Lahiri (2001)). Also this approach is substantially different from ours, since our BGP equilibria are all determinate and are due to the presence of noisy components affecting the research industry costs.

${ }^{5}$ Because of the similarity with our paper and their qualitative results, the seminal work by Evans et al. (1998) deserves some specific comments. Indeed, also Evans et al. (1998) show that under specific conditions a stylized economic growth model may give rise to a low growth trap and a growth cycle in which the economy stochastically switches between periods of low and high growth. However, the underlying argument and the type of dynamics at the basis of their analysis is substantially
} 
(2007)), it also relies upon stochastic growth models in which the source of the shock is completely exogenous (Walde (2005)). Probably, the work most close to ours is Bambi et al. (2014), which analyzes an endogenous growth model with expanding product variety showing that cyclical fluctuations may arise as a result of implementation delays in the innovation process. Despite their setting is quite similar to ours (an almost traditional endogenous growth model) the mechanism underlying output fluctuations is substantially different since we do not allow for time delays but simply for some sort of interaction among firms operating in the research industry. Moreover, different from theirs, our model shows the existence of a growth trap threshold, allowing to clearly distinguish economies which will experience low and high growth rates, which is again simply due to the interaction among research firms.

Our paper is also closely related to the literature on poverty traps. The eventual existence and characteristics of poverty traps have been extensively analyzed in literature since the seminal work by Skiba (1978). Different explanations of why multiplicity of equilibria and thus poverty traps may exist have been put forward, and they include increasing returns and imperfect competition, coordination failure, matching problems and increasing returns (see Azariadis and Stachurski (2005) for an exhaustive survey). However, all these theories proposed thus far outline sources of multiplicity in levels, suggesting thus that under certain conditions an economy may eventually end up in poverty, that is a situation of stagnation with no long run growth. Our model instead suggests the potential existence of equilibrium multiplicity in growth rates, meaning that an economy may eventually end up in a situation of long run growth characterized by low growth rates. In order to distinguish this result from what traditionally discussed in the poverty traps literature we refer to such an outcome as a "low growth trap". To the best of our knowledge, apart from the very recent paper by Agénor and Canuto (2015) in an overlapping generation setting, there is no other study characterizing the eventual existence of low growth traps. The implications of the existence of a low growth trap threshold are however very intuitive and in line with empirical evidence: some countries will experience fast economic growth while others slow economic growth, meaning that income gaps will tend to widen over time characterizing thus a situation of long run divergence, as traditionally found in the empirics on economic growth, especially between developed and developing countries ${ }^{6}$ (Dowrick (1992), Pritchett (1997)). Finally, our model predicts a very important role for economic policy, since in the case of a low growth trap the government, by simply rising the level of taxation on households in order to increase the revenues granted to research firms, may be able to completely solve the trap problem. This does not simply mean that the low growth trap threshold may be exceeded, as the traditional policy implication of poverty trap models (see for example Sachs et al (2004), or more recently La Torre et al. (2015)), but that the threshold itself will cease to exist ensuring thus that the economy is able to experience fast economic growth.

The paper proceeds as follows. Section 2 focuses on the research industry and describes its peculiarities without considering its implications for the whole economy. Specifically, the research industry is populated by a large number of profit-seeking firms facing a dichotomous choice. On the one hand, these firms are heterogeneous in their propensity to innovate, and on the other hand, their decision whether to innovate or not is partly affected by the behavior of other firms in the industry through an externality component. We characterize the research industry dynamics deriving in the infinite dimensional case an explicit expression

different from ours, since, apart from relying on a discrete time setup, the driver of the entire economic dynamics in their model is represented by shocks on agents' expectations which affect the learning dynamics associated with multiple perfect-foresight equilibria. Our results, instead, are derived in a micro-founded model where firm-specific shocks within the research industry, by determining the evolution of technology, propagate in the whole economy eventually generating growth cycles; the concept of endogenous fluctuations we describe is thus not related to either expectational indeterminacy or self-fulfilling growth cycles, which represent the traditional mechanisms discussed in the business cycle literature (Evans et al. (1998); Furukawa (2007)). The fact that such very different setups allow to generate qualitatively similar dynamics suggests that endogenous growth cycles and low growth traps are not only rare theoretical possibility but rather outcomes quite common whenever we depart from the traditional economic growth framework.

${ }^{6}$ Despite the existence of some (absolute) convergence within a small number of industrialized countries (see, for example, Barro and Sala-i-Martin (1995)), convergence clubs represent more the exception rather than the rule in the empirics of economic growth. 
which allows us to describe the (aggregate) behavior of research firm in terms of the share of firms actively engaged in research activities. Section 3 integrates the research industry in a traditional macroeconomic model of endogenous growth, where the government finances research by taxing households, and the overall level of technology in the economy depends on the share of firms engaged in innovation. Section 4 shows that the BGP equilibrium, which strictly depends upon the behavior of research firms, may or may not be unique according to the magnitude of the externality-induced profit component; we also characterize the dynamic properties of different BGP equilibria, identifying the eventual existence of a low growth trap along with its policy implications. In Section 5 we focus on one important implication of the eventual multiplicity in BGP equilibria for the finite dimensional case; we show that when the number of research firms is finite, the probabilistic nature of the model implies that the locally stable equilibria turn out to be metastable: sudden and unpredictable regime switchings among the low and high regimes happen along trajectories, resulting thus in a cycling economic behavior. In Section 6 we propose a generalization of our baseline model in which the incentive to innovation is no longer constant, but it depends on the overall level of technological advancement in the economy; we show that despite the higher degree of sophistication in the model's structure, the results are qualitatively similar to those in its baseline version. In Section 7 we discuss how our model relates to the middle-income trap hypothesis, suggesting that after a first stage of take off characterized by rapid growth developing countries may face a significant growth slowdown; differently from previous research which identify mainly inter-sectoral dynamics as a potential source of growth slowdowns, we argue that this may also be the result of intra-sectoral dynamics (driven by social interactions and technology diffusion) within the research industry. Section 8 presents concluding remarks and proposes directions for future research. Technical details about the rationale behind the random utility approach characterizing research firms' payoff, and the metastability and probabilistic features of the transition times associated with the finite dimensional model are discussed in Appendix $\mathrm{A}$ and $\mathrm{B}$, respectively.

\section{Research Activities and Intra-Industry Interactions}

We consider a research industry populated by a large number of research firms which try to maximize the profits associated with their research activities; specifically, there exist $N$ firms indexed by $i=1, \ldots, N$. For the sake of simplicity we assume that the research choice is just binary, thus we do not try to properly quantify research efforts. Thus, any research firm needs to decide whether to engage in research activities or not, thus it needs to compare the profit it will obtain by performing research with the zero-profit associated with no research activities.

If a firm actively engages in research activities it will give rise with no uncertainty to an innovation, which generates a given (fixed) amount of revenues $h \geq 0$ associated with the sale of the (unitary) innovation 7 . In order to produce one unit of innovation, the firm faces a (stochastic) production cost $z+\zeta_{i}$, where $z \geq 0$ denotes the cost common to all the firms and $\zeta_{i}$ is a random firm-specific shock. Apart from these private components of the profit structure, research profits are also affected by a social component associated with the number of firms actively engaged in research activities. Specifically, the size of the research industry through an externality 8 channel determines whether profits, ceteris paribus, tend to rise or fall. There are two different cases that need to be considered: an increase in the number of firms actively engaged in research may increase the profit for the whole research industry and thus rise the profit of the individual research firm; alternatively, an increase in the number of firms actively engaged in research may decrease the profit

\footnotetext{
${ }^{7}$ For the time being we do not look at the demand side of the innovation market, but this will be introduced in a very stylized way in Section 3 , where we assume that the government buys such an innovation. The amount of revenue $h$ can thus be interpreted as the incentive provided by the government to induce firms to perform research activities, or alternatively as the price at which it purchases the innovation from research firms.

${ }^{8}$ This externality in research profits may be interpreted in terms of the availability of potential trading partners for the innovation, which reflects into a larger or smaller willingness to produce according to the sign of $J$ in (1). With this respect, the market for innovation is similar to the trading market proposed in Diamond $(1982)$.
} 
for the whole research industry and thus lower the profit of the individual research firm. The former case represents the so-called "standing-on-the-shoulder effect", that is innovation by some firms increases the possibility of further innovation by others, while the latter case the "fishing-out effect", that is innovation by some firms decreases the possibility of further innovation by others (Jones (2005)). Formally, we model the individual firm research profits as in random utility models (see Brock \& Durlauf (2001) and Barucci $\&$ Tolotti (2012)). Each firm is thus characterized by its specific innovative attitude $\omega_{i, t} \in\{0 ; 1\}$, where $\omega_{i, t}=1\left(\omega_{i, t}=0\right)$ denotes that firm $i$ is (is not) innovating at time $t$. The decision to engage in research activities to produce innovation is based on the following profit structure:

$$
\pi_{i}\left(\omega_{i}\right)=\omega_{i}\left[h-\left(z+\zeta_{i}\right)+J\left(\tilde{x}_{i}^{e}-\frac{1}{2}\right)\right] .
$$

If the firm does not innovate $\left(\omega_{i, t}=0\right)$ the profit above is simply null, $\pi_{i}(0)=0$. If the firm does innovate $\left(\omega_{i, t}=1\right)$ the profit is equal to $\pi_{i}(1)=\left[h-\left(z+\zeta_{i}\right)+J\left(\tilde{x}_{i}^{e}-1 / 2\right)\right]$, where the first two terms represent the private component of profit while the third term is the social component related to the effect of externalities. The impact of the research externality is equal to $J\left(\tilde{x}_{i}^{e}-1 / 2\right)$, where $J \in \mathbb{R}$ determines the sign and the magnitude of the externality effect and $\tilde{x}_{i}^{e}$ is the expectation of firm $i$ about the average of the choices of other firms: $\tilde{x}_{i}^{e}=\frac{1}{N-1} \mathbb{E}\left[\sum_{j \neq i} \omega_{j}\right]$. Note that the sign of $J$ determines the type of externality affecting research firms: whenever $J>0$ individual profits tend to increase as a result of the research performed by others (standing-on-the-shoulder effect), while whenever $J<0$ individual profits tend to fall (fishing-out effect). The term $\left(\tilde{x}_{i}^{e}-1 / 2\right)$ states that in quantifying the impact of the (positive or negative) externalityinduced profit component firms look at what the majority of other firms does. Indeed, the term $1 / 2$ refers exactly to one half of the total population of research firms, thus if $\tilde{x}_{i}^{e}>1 / 2$ then firm $i$ will expect more than half of the firms to do research. Finally, the random components of cost, $\zeta_{i}, i=1, \ldots, N$ are i.i.d. random shocks drawn from a common distribution $\eta$, which affect with different intensity the perceived profit of individual firms. Two remarks on the profit structure are needed. First of all, note that the profit $\pi$ depends on the subjective expectation of the firm about others' actions. With this respect, it can be seen as the realized profit once conditioned on agent's expectation about others' actions. Secondly, the random component of the profit is entirely related to the cost structure. We could in principle build a profit structure where randomness may inpact jointly or separately both revenues and costs. Besides amounting in a more complicated probabilistic structure, this woud not have any significant qualitative implications. For a more comprehensive discussion about the rationale behind the profit structure as in (1), we refer the reader to Appendix A.

It can be easily verified that profits as in (1) turn into a probabilistic choice model where:

$$
\mathbb{P}\left(\omega_{i}=1 \mid \tilde{x}_{i}^{e}\right)=\eta\left[h-z+J\left(\tilde{x}_{i}^{e}-\frac{1}{2}\right)\right] .
$$

As shown in the literature on social interactions (see Blume \& Durlauf (2003)), a dynamic counterpart of such a model can be derived. Define

$$
x_{t}^{N}=\frac{1}{N} \sum_{i=1}^{N} \omega_{i, t}
$$

as the fraction of innovative firms at time $t$ and assume this quantity is observable; we refer to $x_{t}^{N}$ as the "innovation share". Similarly as in the static model, we assume that firms can decide whether to invest or not at any time $t$ by considering its potential revenue $h$, cost $z$ and the current value of the innovation share $\widetilde{x}_{i}^{e}$. Indeed,

$$
\mathbb{P}\left(\omega_{i, t+\Delta t}=1 \mid \omega_{i, t}, x_{t}^{N}\right)=\eta\left[h-z+J\left(x_{t}^{N}-\frac{1}{2}\right)\right] .
$$

It turns out that the Markovian dynamics induced by (4) are difficult to study in the finite dimensional population model; nevertheless, it is possible to describe in closed-form the (deterministic) dynamics emerging from the asymptotic system when letting the number of research firms go to infinity. In particular, the 
following result describes the time evolution of $x_{t}$ which is the fraction of innovative firms at time $t$ when we let $N \rightarrow \infty$. To this aim, we assume that the shocks $\zeta_{i}$ follow a centered logistic distribution $?^{9}$ with parameter $\beta>0$ :

$$
\eta(x)=\mathbb{P}\left(\zeta_{i} \leq x\right)=\frac{1}{1+e^{-\beta x}} .
$$

In this context, $\beta$ is a measure of the dispersion of opinion in the population of firms: $\beta=0$ would represent a situation in which the firms decide to innovate or not by tossing a coin; on the contrary, $\beta \rightarrow \infty$ would mean that the firms do not receive any stochastic signal (i.e., the random cost component) and decide just by looking at the sign of $h-z+J\left(x_{t}^{N}-\frac{1}{2}\right)$. In the next proposition, we provide a law of large numbers ${ }^{10}$ for the stochastic process $x_{t}^{N}$, showing that it converges to a limiting (deterministic) process $x_{t}$, whose law of motion is described by a suitable differential equation characterizing the (deterministic) evolution of the share of innovative firms.

Proposition 1. Let $x_{t}^{N}=\frac{1}{N} \sum_{i=1}^{N} \omega_{i, t}$ be the share of innovative firms at time $t$. Suppose $\lim _{N \rightarrow \infty} x_{0}^{N}=x_{0}$. Then, when $N \rightarrow \infty$, the family of stochastic processes $\left(x^{N}\right)_{N \geq 0}$, where $x^{N}:=\left(x_{t}^{N}\right)_{t \geq 0}$, converges almost surely to $x:=\left(x_{t}\right)_{t \geq 0}$, where $x_{t}$ solves

$$
\dot{x}_{t}=\frac{1}{2} \tanh \left\{\beta\left[h-z+J\left(x_{t}-\frac{1}{2}\right)\right]\right\}-x_{t}+\frac{1}{2},
$$

for a given initial condition $x_{0}$.

Proof. We can recover the standard Blume \& Durlauf (2003) framework by rearranging the state variables to take values on $\{-1 ;+1\}$. Define $\zeta_{i}=1$ when $\omega_{i}=1$ and $\zeta_{i}=-1$ when $\omega_{i}=0$. In this case, we have that

$$
\begin{array}{r}
\mathbb{P}\left(\zeta_{i, t}=1 \mid \zeta_{i, t}, m_{t}^{N}\right)=\eta\left(h-z+J / 2 \cdot m^{N}(t)\right), \\
\mathbb{P}\left(\zeta_{i, t}=-1 \mid \zeta_{i, t}, m_{t}^{N}\right)=1-\eta\left(h-z+J / 2 \cdot m^{N}(t)\right),
\end{array}
$$

where now $m^{N}(t)=\frac{1}{N} \sum_{i} \zeta_{i, t}$ takes values on [-1,1]. Arguing similarly as in Barucci \& Tolotti (2012), it can be shown that, under the assumptions of Proposition 1 .

$$
\lim _{N \rightarrow \infty} m_{t}^{N}=m_{t}
$$

where $m_{t}$ is the unique solution to

$$
\dot{m}_{t}=\tanh \left\{\beta\left(h-z+J \cdot \frac{m_{t}}{2}\right)\right\}-m_{t} ; \quad m_{0}=2 x_{0}-1 .
$$

Since $x_{t}=\frac{m_{t}+1}{2}$, equation (5) immediately follows.

Proposition 1 allows to approximate the random dynamic behavior of research firms through a deterministic equation which provides us with a simple but useful benchmark to characterize the outcome in the research industry. This allows us to analytically derive the outcome in the approximated deterministic version of the model that we shall introduce in a while and compare this with the "true outcome" in its stochastic version. Note, moreover that in order to derive the approximated dynamic equation (5), random shocks play an essential role in generating heterogeneity in research firms' behavior and thus in giving rise

\footnotetext{
${ }^{9}$ We could in principle use any continuous probability distribution. The logistic is vastly used in the context of random utility models. One reason being that the dynamics obtained under this assumption have a logistic shape which seems to represent patterns underlying many social phenomena (see Anderson et al. (1992)).

${ }^{10}$ We provide here a straightforward proof based on the argument developed in Blume \& Durlauf (2003). A more detailed and alternative proof of the law of large numbers will be provided in Section 6 in a more general setting. Note that in that case, we can only provide a weak convergence result, being the proof based on the convergence of generators of the underlying Markov processes.
} 
to potentially nontrivial outcomes (see Appendix A for further details). In the following, we shall restrict our analysis to the nontrivial situation in which random shocks do affect firms' decision and thus firms are effectively heterogeneous in their propensity to innovate. In such a framework, the quantity $x_{t}$ characterizes the (approximated) fraction of innovative firms in a large economy of research firms subject to externalities and private signals. Since (5) provides us with an explicit expression for describing the behavior of research firms, as we shall see in the next section, it is now straightforward to incorporate the research industry in a canonical endogenous growth model. This allows us to understand to what extent the presence of firm interactions in the research industry is going to affect the macroeconomic outcome, further distinguishing between the standing-on-the-shoulders and the fishing-out cases. Since the role of the fixed cost $z$ is negligible in our setting, for the sake of simplicity in the remainder we will set it equal to zero.

\section{The Macroeconomic Model}

Apart from the characterization of the research market which to some extent resembles what discussed in Marchese at al. (2014), the model is an almost standard endogenous growth model characterized by households, productive and research firms, and a government. Households try to maximize their lifetime welfare, by determining how much to consume given the dynamic evolution of capital. Productive firms produce competitively the unique final consumption good, by determining how many workers and how much capital to employ given the available technology. Research firms determine whether to invest or not in innovation, and overall technological progress depends on the share of research firms which actively engage in research activities. The government aiming at maintaining a balanced budget at any point in time levies taxes on households to finance such research activities. Households and productive firms are homogeneous, thus we analyze their behavior as traditional representative agents. Research firms are instead heterogeneous in their propensity to innovate, and their behavior is consistent with what discussed in the previous section.

The representative household's problem consists of maximizing its welfare given its initial capital endowment $k_{0}$ and the law of motion of capital, $k_{t}$, by choosing how much to consume, $c_{t}$, and supplying inelastically labor. The household size, $L$, is constant and it is assumed to be infinitely large. Welfare is defined according to the average utilitarian criterion 41 , thus it is equal to the infinite discounted sum ( $\rho$ is the pure rate of time preference) of instantaneous utilities, which depend solely upon consumption. The instantaneous utility function is assumed to take the following isoelastic form: $u\left(c_{t}\right)=\frac{c_{t}^{1-\sigma}-1}{1-\sigma}$, where $\sigma>1$ is the inverse of the intertemporal elasticity of substitution. As usual lowercase letters denote per capita variables while uppercase letters aggregate variables. The household's problem in per capita terms can be written as:

$$
\begin{array}{ll}
\max _{c_{t}} & W=\int_{0}^{\infty} \frac{c_{t}^{1-\sigma}-1}{1-\sigma} e^{-\rho t} d t \\
\text { s.t. } & \dot{k}_{t}=\left(1-\tau_{t}\right)\left(r_{t} k_{t}+w_{t}\right)-c_{t},
\end{array}
$$

where $r_{t}$ is the capital rental rate, $w_{t}$ the wage rate and $\tau_{t}$ a (time-varying) income tax rate. The first terms in the RHS of (8) represent the disposable income which needs to be allocated between consumption $\left(c_{t}\right)$ and capital investments $\left(\dot{k}_{t}\right)$.

Output is produced by competitive productive firms according to a Cobb-Douglas production function, combining labor, $L$ (inelastically supplied by households), and capital, $K_{t}$. The production function in per

\footnotetext{
${ }^{11}$ Note that since household size is constant, in our model the difference between welfare as defined according to either the average or total utilitarian criterion is simply a constant, equal to household size (see Marsiglio (2014) for a recent discussion of the implications of average and total utilitarianism on economic growth). However, since the size of household is assumed infinitely large (why this is needed will become clear later) we cannot rely on total utilitarianism since this would imply that household's objective function is infinite.
} 
capita terms takes the following form:

$$
y_{t}=A_{t} k_{t}^{\alpha}
$$

where $\alpha \in(0,1)$ is the capital share while $A_{t}$ a technological factor, representing total factor productivity. Productive firms take the level of technology as given and maximize their instantaneous profits, determining thus the rental rate of capital, $r_{t}$ (and the wage rate, $w_{t}$ ).

Research firms indexed by $i=1, \ldots, N$ are heterogeneous in their propensity to innovate $\omega_{i, t}$ and try to maximize the profits associated with their research activities. Their behavior is identical to what discussed in the previous section, thus is determined by the comparison between their profit when innovating $\left(\omega_{i, t}=1\right)$ and when not $\left(\omega_{i, t}=0\right)$. Whenever innovating they will sell their innovation at a price $\tilde{h}_{t}=h y_{t}$ to the government 12 which does somehow finance the research activities in the overall economy. We assume the number of research firms is infinitely large such that Proposition 1 holds.

The government, by taxing households, collects a tax revenue $\tau_{t} y_{t}$ from each household, which is used to buy innovations at a price $\tilde{h}_{t}$ (in units of output) from each research firm actively engaged in research activities, $\sum_{i} \omega_{i}$. In order to maintain a balanced budget at any point in time, the government budget constraint reads as $\tau_{t} y_{t} L=h y_{t} \sum_{i} \omega_{i, t}$, implying that $\tau_{t} \frac{L}{N}=h \frac{\sum_{i} \omega_{i, t}}{N}$. Since the number or research firms $N$ is infinitely large the previous equation can be non-trivially verified only if the number of households $L$ is infinitely large as well, such that the household to research firm ratio $\ell=\lim _{(L, N) \rightarrow(\infty, \infty)} \frac{L}{N}>0$ is constant and finite. Provided that both the number of households and research firms are infinitely large, the budget constraint can be rewritten as follows:

$$
\tau_{t} \ell=h x_{t}
$$

where $x_{t}$ is the share of innovative firms whose dynamics is given in (5). Once an innovation is bought by the government, it is immediately released in the public domain to allow productive firms to use such an innovation for free to produce the final consumption good (Marchese at al. (2014)). This means that the government plays an essential role by spreading innovations in the economy by buying them from firms, solving thus an important coordination problem. This is consistent with recent evidence suggesting that most technological advances have effectively been made possible by entrepreneurial activities pursued by governments (Mazzucato $(\overline{2013}))$.

By financing research activities the government determines the time evolution of the total factors productivity. Indeed, the overall level of technology is determined by the interaction among research firms. Specifically, we assume that it evolves according to the following law of motion:

$$
\dot{A}_{t}=\phi x_{t} A_{t},
$$

where $\phi>0$ is a scale parameter and $x_{t}$ represents the share of research firms which actively engage in innovative activities. According to (11) for technological progress to occur it does not matter the size of the research industry (i.e., how many research firms exist) but the relative size of innovative firms with respect to the industry. If none does research $\left(x_{t}=0\right)$ then technological progress does not occur, while if all firms do research $\left(x_{t}=1\right)$ then technological progress occurs at a strictly positive rate $\phi$. For any situation different from these two extreme cases, the rate of technological progress will lie between 0 and $\phi$; which specific rate will arise depends on the behavior of research firms and their interaction within the research industry.

\footnotetext{
${ }^{12}$ Note that in the research firms' profit structure (1), only the constant term $h$ appears. We, in fact, use a "per unit" measure of (perceived) profit $h=\tilde{h}_{t} / y_{t}$. Such a measure is more appropriate to study firms interaction since $\tilde{h}_{t}$ diverges to infinity over time exactly as $y_{t}$. Although still tractable (see Example 6.2, the formulation with the "non-discounted" $\tilde{h}_{t}$ turns out to be trivial and thus less interesting, since the private profit component (related to $\tilde{h}_{t}$ ) is not comparable with the social component (which is bounded). See Section 6, where we provide some examples related to the more general case of a time-varying $h_{t}$, including also the non-discounted $\hat{h}_{t}$ case.
} 
In general equilibrium, all agents maximize their objective function and all markets clear. The economy is completely characterized by the following system of differential equations and the given initial conditions $k_{0}, x_{0}$ and $A_{0}$ :

$$
\begin{aligned}
& \frac{\dot{c}_{t}}{c_{t}}=\frac{1}{\sigma}\left[\left(1-\frac{h}{\ell} x_{t}\right) \alpha A_{t} k_{t}^{\alpha-1}-\rho\right] \\
& \dot{k}_{t}=\left(1-\frac{h}{\ell} x_{t}\right) A_{t} k_{t}^{\alpha}-c_{t} \\
& \dot{x}_{t}=\frac{1}{2} \tanh \left\{\beta\left[h+J\left(x_{t}-\frac{1}{2}\right)\right]\right\}-x_{t}+\frac{1}{2} \\
& \dot{A}_{t}=\phi x_{t} A_{t}
\end{aligned}
$$

Note from the above equations that the research industry dynamics turns out to be independent from other macroeconomic variables. Despite this might seem a strong limitation of our model, as we shall see in Section 6, results will not be qualitative different even in a more sophisticated formulation in which research industry and macroeconomic outcomes affect each other. It seems convenient thus to present the model first in its simplest possible form. Apart from the case in which $x_{t}$ converges to zero (which however will never be an equilibrium), the above system (12), (13), (14) and (15) is not stationary (i.e., it does not show any equilibrium at all), thus in order to study its dynamic behavior it may be convenient to recast the system in a stationary system as traditionally done in the endogenous growth literature. From the equilibrium properties of this latter system, we will then be able to infer the properties of the BGP equilibrium associated with (12), (13), (14) and (15). A BGP equilibrium denotes a situation in which all variables grow at a constant (possibly non-negative) rate, and deriving and discussing the characteristics of the BGP equilibrium is our main goal in next section.

\section{BGP Equilibrium}

By introducing the variables $\chi_{t}=\frac{c_{t}}{k_{t}}$ and $\varphi_{t}=A_{t} k_{t}^{\alpha-1}$, denoting the consumption to capital ratio and the average product of capital respectively, it is possible to recast the above system in the following stationary system:

$$
\begin{aligned}
\frac{\dot{\chi}_{t}}{\chi_{t}} & =\chi_{t}-\frac{\rho}{\sigma}-\frac{\sigma-\alpha}{\sigma}\left(1-\frac{h}{\ell} x_{t}\right) \varphi_{t} \\
\frac{\dot{\varphi}_{t}}{\varphi_{t}} & =\phi x_{t}-(1-\alpha)\left(1-\frac{h}{\ell} x_{t}\right) \varphi_{t}+(1-\alpha) \chi_{t} \\
\dot{x}_{t} & =\frac{1}{2} \tanh \left\{\beta\left[h+J\left(x_{t}-\frac{1}{2}\right)\right]\right\}-x_{t}+\frac{1}{2}
\end{aligned}
$$

At equilibrium the above system is characterized by the following steady state values:

$$
\begin{aligned}
\bar{\chi} & =\frac{(1-\alpha) \rho+(\sigma-\alpha) \phi \bar{x}}{\alpha(1-\alpha)} \\
\bar{\varphi} & =\frac{(1-\alpha) \rho+\sigma \phi \bar{x}}{\alpha(1-\alpha)\left(1-\frac{h}{\ell} \bar{x}\right)} \\
\bar{x} & =\frac{1}{2} \tanh \left\{\beta\left[h+J\left(\bar{x}-\frac{1}{2}\right)\right]\right\}+\frac{1}{2}
\end{aligned}
$$

where $\bar{x}$ cannot be determined explicitly. However since $x_{t} \in[0,1]$ it follows that $\bar{x}$ will always be nonnegative. This means that provided that $\ell>h$, the steady state values $\bar{\chi}$ and $\bar{\varphi}$ will be strictly positive. We summarize the results about the BGP equilibria and their stability in the following proposition. 
Proposition 2. Assume $\ell>h$; then along a BGP equilibrium, the economic growth rate, $\gamma$, is strictly positive and given by the following expression:

$$
\gamma \equiv \gamma_{c}=\gamma_{k}=\frac{\gamma_{A}}{1-\alpha}=\gamma_{y}=\frac{\phi \bar{x}}{1-\alpha}>0
$$

where $\bar{x}$ denotes the steady state value of $x_{t}$. Moreover, there exist two positive threshold levels, $J^{t}(\beta)$ and $h^{t}(J, \beta)$, given by the following expressions

$$
\begin{aligned}
J^{t}(\beta) & =\frac{2}{\beta} \\
h^{t}(J, \beta) & =\frac{J}{2} \sqrt{\frac{\beta J-2}{\beta J}}+\frac{1}{\beta} \ln \left(\sqrt{\frac{\beta J}{2}}-\sqrt{\frac{\beta J-2}{2}}\right),
\end{aligned}
$$

such that:

i) if $J<J^{t}(\beta)$, there exists a unique $\gamma^{*}$ and the unique BGP equilibrium is saddle-point stable with a two-dimensional stable manifold;

ii) if $J>J^{t}(\beta)$, then two alternative outcomes are possible:

a) if $h>h^{t}(J, \beta)$, there exists a unique $\gamma^{*}$ and the unique BGP equilibrium is saddle-point stable with a two-dimensional stable manifold;

b) if $h<h^{t}(J, \beta)$, there exist three BGP equilibria corresponding to three values $\gamma_{L}<\gamma_{M}<\gamma_{H}$. The intermediate one is saddle-point stable with a one-dimensional stable manifold, whereas the two extreme ones are (locally) saddle-point stable, each with a two-dimensional stable manifold.

Proof. By plugging the steady state values of $\chi_{t}$ and $\varphi_{t}$ back in the original equations (12)-(15), it is straightforward to derive the BGP growth rate $\gamma$, as in (22). The characteristics of $\gamma$ strictly mimic those of $\bar{x}$. Indeed, multiplicity is due to the possible multiplicity of the steady states of equation (5). As already shown in the literature (see Brock \& Durlauf (2001)), it turns out that, depending on the values of the parameters, we can have a unique stable equilibrium $(\bar{x})$ for $(5)$ or three equilibria $\left(\bar{x}_{L}<\bar{x}_{M}<\bar{x}_{H}\right)$, two of which are locally stable $\left(\bar{x}_{L}\right.$ and $\left.\bar{x}_{H}\right)$. A similar threshold value for $J$, equal to $1 / \beta$, is also derived by Brock $\&$ Durlauf (2001); note that the factor 2, appearing in our statement, depends on the transformation from the variable $m_{t}$ to the rescaled variable $x_{t}$ as shown in the proof of Proposition 1 .

Concerning the value of $h^{t}$, in Olivieri and Vares (2005) (see Sections 4.1.1. and 4.3) it is shown that the fixed point problem $m=\tanh (\tilde{\beta}(m+\tilde{h}))$ admits multiple solutions as soon as $\tilde{\beta}>1$ and $\tilde{h}<$ $\sqrt{\frac{\tilde{\beta}-1}{\tilde{\beta}}}+\frac{1}{\tilde{\beta}} \ln (\sqrt{\tilde{\beta}}-\sqrt{\tilde{\beta}-1})$. According to $(6)$ and assuming $z=0$ without loss of generality, we can rewrite our equation in $x$ in the form $m=\tanh \left(\beta h+\frac{\beta J}{2} m\right)$. Therefore, $h^{t}$ is derived from the above expressions by setting $\tilde{\beta}=\beta J / 2$ and $\tilde{h}=2 h / J$. From $(22)$, if there are multiple equilibria for $x$, then the system admits multiple equilibria as well.

Concerning stability, by linearization around a steady state it is possible to analyze the (local) stability properties of the above system by deriving the following Jacobian matrix:

$$
J(\bar{\chi}, \bar{\varphi}, \bar{x})=\left[\begin{array}{ccc}
\bar{\chi} & -\frac{\sigma-\alpha}{\sigma}\left(1-\frac{h}{\ell} \bar{x}\right) \bar{\chi} & \frac{\sigma-\alpha}{\sigma} \frac{h}{\ell} \bar{\varphi} \bar{\chi} \\
(1-\alpha) \bar{\varphi} & -(1-\alpha)\left(1-\frac{h}{\ell} \bar{x}\right) \bar{\varphi} & \phi \bar{\varphi}+(1-\alpha) \frac{h}{\ell} \bar{\varphi}^{2} \\
0 & 0 & \Lambda
\end{array}\right],
$$

where $\Lambda=\left.\frac{\partial \dot{x}_{t}}{\partial x_{t}}\right|_{x_{t}=\bar{x}}$. It is straightforward to show that the eigenvalues are given by the following expressions $\lambda_{1}=\Lambda$, and $\lambda_{2,3}=\frac{\Delta \pm \sqrt{\Delta^{2}+\Theta}}{2}$, where $\Delta=\bar{\chi}-(1-\alpha)\left(1-\frac{h}{\ell} \bar{x}\right) \bar{\varphi}>0$ and $\Theta=4 \frac{\alpha}{\sigma}(1-\alpha)\left(1-\frac{h}{\ell} \bar{x}\right) \bar{\chi} \bar{\varphi}>0$, from which it directly follows that $\lambda_{2}>0$ and $\lambda_{3}<0$. Independently of what the sign of $\Lambda$ is, there exists at least 
one positive and one negative eigenvalue, thus any possible equilibrium is saddle-point stable. Moreover, it is possible to show that $\Lambda<0$ for $\bar{x}_{L}$ and $\bar{x}_{H}$ and $\Lambda>0$ for $\bar{x}_{M}$. Therefore, the stable manifold associated with the three equilibria has dimension 2 for $\gamma_{L}$ and $\gamma_{H}$ and dimension one for $\gamma_{M}$.

The parameter condition required by Proposition 2 is needed in order to ensure that the BGP equilibrium is well defined. Intuitively, it requires that the household to research firm ratio $(\ell)$ is large enough to provide the government with the resources needed to promote research activities $(h)$. Along a BGP the economic growth rate $\gamma$ depends negatively on $\alpha$ and positively on $\phi$ and, more importantly on the equilibrium share of innovative firms $\bar{x}$. This means that our model economy does not show any scale effect, since the growth rate is independent of any aggregate variable ${ }^{13}$. However, since the equilibrium share of research firms may not be unique ${ }^{14}$, also the BGP equilibrium turns out to be not unique, and this is strictly related to the size of the externality parameter, $J$. Indeed, Proposition 2 suggests that in the fishing-out case $(J<0)$ there always exists a unique saddle-point stable BGP equilibrium; however, in the standing-on-the-shoulder case $(J>0)$ there is a richer variety of possible outcomes. Whenever the standing-on-the-shoulder effect is weak (i.e., the magnitude of the positive externality is small) a unique stable equilibrium will emerge. In the case of a sufficiently large externality, then the number of equilibria depends on the value of the incentive mechanism provided by the amount of revenues obtained, $h$. A large $h$ makes the equilibrium unique, whereas, a small $h$ gives rise to the presence of two locally stable equilibria15. As a matter of expositional simplicity, in the following we will refer to the case (i) in Proposition 2 as the "small externality case" and to the case (ii) as the "large externality case". Note that the macroeconomic behavior closely resembles the behavior on the innovation share, and when the equilibrium innovation share is unique (multiple) then the BGP equilibrium is unique (multiple) as well. Specifically, in the case of multiple equilibria, if $x_{0}<\bar{x}_{M}$ then $x_{t}$ will converge to $\bar{x}_{L}$ (and the BGP growth rate will be low, $\gamma_{L}$ ), while if $x_{0}>\bar{x}_{M}$ then $\bar{x}_{H}$ will be reached instead (and the BGP growth rate will be high, $\gamma_{H}$ ). Thus, the initial fraction of innovative firms plays a crucial role in determining which BGP equilibrium will be effectively achieved ${ }^{16}$.

In order to understand more in depth what are the characteristics of the BGP equilibrium, we now analyze the behavior of the economy under a realistic model's parametrization. Specifically, we set the inverse of the intertemporal elasticity of substitution, $\sigma$, equal to 2, the rate of time preference, $\rho$, to 0.04 , the capital share, $\alpha$ to 0.33 (Mullingan and Sala-i-Martin (1993)); the scale parameter determining the rate of growth of technology, $\phi$ is calibrated to 0.04 , in order to obtain an economic growth rate equal to 0.03 (in the case in which the equilibrium share of innovative firms is exactly equal to one half); the other parameter values, are set arbitrarily in order to make sure that the assumption required in Proposition 2 is met and that our qualitative results are as clear as possible. We thus set the households to research firm ratio, $\ell$, equal to 1,000 , the measure of the dispersion of opinion in the population of research firms, $\beta$ equal to 1 , while we let the revenue provided to research firms, $h$, and the size of the externality parameter, $J$, vary in order to see how they affect the BGP economic growth rate $\gamma$. Table 1 summarizes the parameter values employed in our analysis.

In Figure 1 we show how the BGP growth rate $\gamma$ varies for different values of the externality parameter, $J$,

\footnotetext{
${ }^{13} \mathrm{An}$ increase in the number of firms in the research industry does not rise the overall economic growth rate. This rate can increase only if the equilibrium share of innovative firms rises.

${ }^{14}$ Note that the eventual multiplicity in the equilibrium of the innovation share is due to the heterogeneity in research firms. As more specifically discussed in Appendix A, in the case of homogeneous research firms the equilibrium innovation share will necessarily be unique and equal to either zero or one, meaning that the BGP growth rate will be either null or maximal, respectively. Such an outcome is clearly possible but also trivial, thus in our discussion we focus only on the most interesting case in which research firms are heterogeneous.

${ }^{15}$ Note that the intermediate equilibrium $\gamma_{M}$, although saddle point stable, is derived from an innovation share $\bar{x}_{M}$ which is linearly unstable on its own. Therefore, unless we assume that the economy is exactly tuned on $x_{0}=\bar{x}_{M}$, this equilibrium will never emerge. For this reason, we will not consider it as a possible realist economic outcome.

${ }^{16}$ The importance of the initial share of innovative firms for the model's outcome is further discussed in Section 5 where we focus on the finite-number of research firms case. We will show that in such a (stochastic) framework the presence of multiple equilibria might give rise to growth cycles.
} 


\begin{tabular}{|c|c|c|c|c|c|}
\hline$\sigma$ & $\rho$ & $\alpha$ & $\ell$ & $\phi$ & $\beta$ \\
\hline 2 & 0.04 & 0.33 & 1,000 & 0.04 & 1 \\
\hline
\end{tabular}

Table 1: Parameter values employed in our simulation.

whenever the revenue parameter, $h$ is set equal to 0 . As expected from Proposition 2, for negative and positive but small enough values of the externality parameter a unique BGP and thus a unique economic growth rate, $\gamma^{*}=\frac{\phi \bar{x}^{*}}{1-\alpha}$ (equal to 0.03), exists. For larger values, three equilibria, namely $\gamma_{L}=\frac{\phi \bar{x}_{L}}{1-\alpha}, \gamma_{M}=\frac{\phi \bar{x}_{M}}{1-\alpha}$ and $\gamma_{H}=\frac{\phi \bar{x}_{H}}{1-\alpha}$ with $\gamma_{L}<\gamma_{M}<\gamma_{H}$, exist and the gap between the high and low economic growth rate, $\gamma_{H}-\gamma_{L}$ rises with $J$.

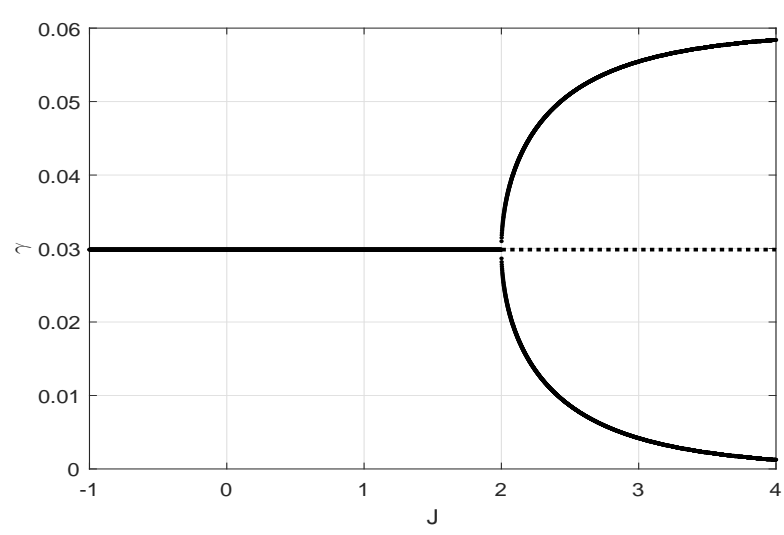

Figure 1: Changes in the economic growth rate, $\gamma$, for different values of the externality parameter, $J$ (revenue parameter, $h$ set equal to 0 ).

Since the existence of either a unique or multiple BGP equilibrium is related to the size of the externality parameter, it may be convenient to separately analyze the cases in which the externality parameter is either small or large. In Figure 2 we thus consider two alternative values $J=1.9$ and $J=2.5$, lying below and above the threshold value $J^{t}=2$ (see Figure 1) respectively; and show how the BGP growth rate $\gamma$ varies with the revenue parameter, $h$. As discussed above, the small externality case represents a situation in which the research sector is characterized by either fishing-out $(J<0)$ or weak standing-on-the-shoulder $\left(0<J \leq J^{t}\right)$ effects. In both the cases, equation 18$)$ shows a unique stable equilibrium and consequently the BGP equilibrium is unique as well: $\gamma^{*}=\frac{\phi \bar{x}^{*}}{1-\alpha}$. The convergence to the steady state of the system $(16)$ - (18) will occur along a two-dimensional stable manifold. We can see that the unique economic growth rate increases with $h$, thus the higher the incentive for research firms to engage in research activities the faster the economic growth (Figure 2, left panel). The large externality case represents instead a situation in which the research sector is characterized by a strong standing-on-the-shoulder $\left(J>J^{t}\right)$ effect. In this case, equation (18) shows three equilibria $\left(\bar{x}_{L}<\bar{x}_{M}<\bar{x}_{H}\right)$, two of which are locally stable $\left(\bar{x}_{L}\right.$ and $\left.\bar{x}_{H}\right)$. As a consequence, the BGP equilibrium is not unique as well: we need to distinguish three BGP equilibria, characterized by an economic growth rate equal to $\gamma_{L}=\frac{\phi \bar{x}_{L}}{1-\alpha}, \gamma_{M}=\frac{\phi \bar{x}_{M}}{1-\alpha}$ and $\gamma_{H}=\frac{\phi \bar{x}_{H}}{1-\alpha}$ with $\gamma_{L}<\gamma_{M}<\gamma_{H}$, respectively. As seen from Proposition 2, the convergence to such three steady states of the system (16) (18) will occur either along a two-dimensional stable manifold (for $\gamma_{L}$ and $\gamma_{H}$ ) or along a one-dimensional stable manifold (for $\gamma_{M}$ ). We can see that the high and low economic growth rate, $\gamma_{H}$ and $\gamma_{L}$ increase with $h$, while the medium one $\gamma_{M}$ falls with $h$; thus the higher the incentive for research firms to engage in research activities the faster the economic growth in each of the two stable equilibria (Figure 2, right panel). The threshold value for $h$ provided by Proposition 2 (and confirmed by our numerical simulation) is $h^{t} \approx 0.078$; only whenever $h<h^{t}$, three equilibria exist. 

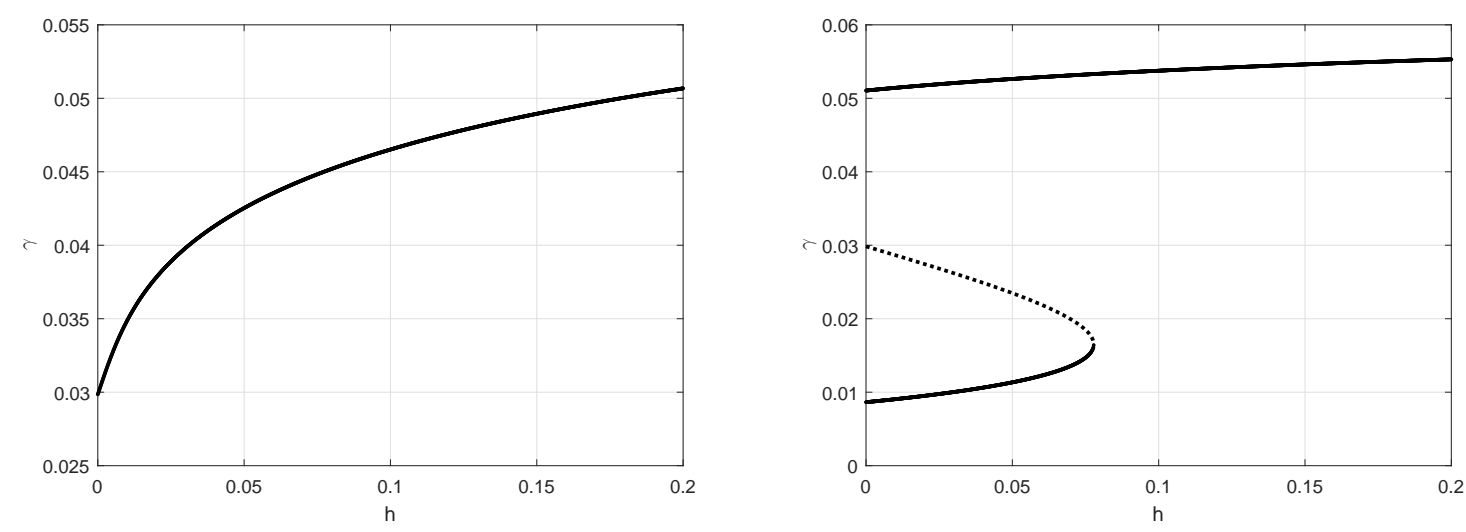

Figure 2: Changes in the growth rate, $\gamma$, for different values of the revenue parameter $h(0 \leq h \leq 0.2)$; the externality parameter $J$ set equal to either 1.9 (left panel) or 2.5 (right panel).

Figure 2 suggests some interesting policy implications, since it clearly shows how the revenue parameter impacts on the equilibrium economic growth rate. Indeed, in the large externality case whenever the revenue provided to research firms is small $\left(h<h^{t}\right)$, three different BGP equilibria exist, and this is strictly related to the existence of three different equilibrium values for the innovation share. Therefore, the same economy may experience different growth rates according to how many research firms actively engage in research activities: if this share is small the economic growth rate will be low while if it is large the economic growth rate will be high. This means that the economy is potentially faced with a low growth trap, which may condemn it to grow slower than what it could potentially do. In such a framework it is natural to wonder what policymakers can do in order to deal with this problem. As traditionally discussed mainly in the context of poverty traps (Sachs et al (2004)), an economy may escape its low growth trap by increasing the innovation share, allowing thus the initial share of research firms $\left(x_{0}\right)$ to exceed its unstable middle equilibrium $\left(\bar{x}_{M}\right)$. Such an outcome might be implemented by simply opening the economy to international trade and providing some incentive for foreign firms actively engaged in research activities to operate also on the domestic market; research activities at international level may thus provide the economy with the push it needs to achieve fast economic growth. However, policymakers may do much more than this, since they can effectively allow the economy not only to escape its low growth trap, but to even solve completely the trap problem. Indeed, by rising enough the revenue provided to each research firm such that $h>h^{t}$, the innovation share will naturally converge towards its unique (higher) equilibrium value; the economic growth rate at equilibrium will be high, and thus the economy will not be trapped into a low growth equilibrium. Such an outcome can be easily implemented by increasing the tax rate applied to households' income in order to finance the increase in the revenue parameter. Indeed, in our model's parametrization the tax parameter $\hat{\tau}$ needed to escape the low growth trap is $\hat{\tau}=\frac{h}{\ell} \bar{x} \approx 0.0413 \%$. The result should be clear from Figure 2 it can also be seen from Figure 3 where we plot the equilibrium values of $\bar{x}$ for two different values of the revenue parameter $h$. This clearly show that with a higher $h$ a unique equilibrium $\bar{x}$ (and thus also a unique BGP) may exist.

\section{$5 \quad$ Metastability and Endogenous Cycles}

As already stressed in Section 2 , the infinite dimensional research market obtained by letting $N$ go to infinity is just a deterministic approximation of the more complex finite $N$ dimensional heterogeneous research industry described by equations (1) - (4). We now focus on some important implications of the fact that the "true" model is actually not deterministic but stochastic since characterized by some intrinsic randomness. 

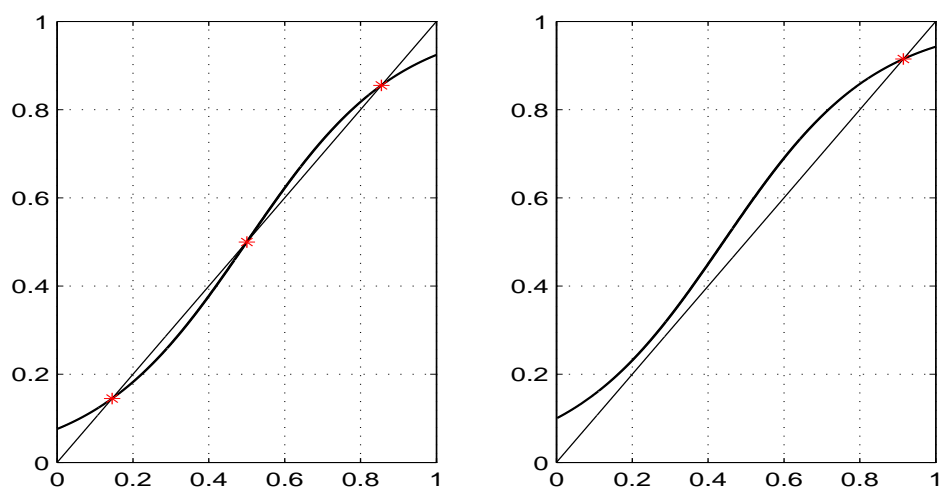

Figure 3: Equilibrium values $\bar{x} \in[0,1]$ (marked with a star) found as the intersection between the bisector line and $f(x)=\frac{1}{2} \tanh \left\{\beta\left[h+J\left(x-\frac{1}{2}\right)\right]\right\}+\frac{1}{2}$ (see equation (21)). Parameters values: $h=0$ (left panel) and $h=0.15$ (right panel), with $J=2.5$.

Indeed, when multiple BPG equilibria exsit:17, the finite dimensional system described in Section 2 has the remarkable property to exhibit a "metastable behavior". As discussed in Mathieu and Picco (1998), a probabilistic system exhibits a metastable behavior when it remains for long times close to an apparent equilibrium, called metastable, and then it suddenly shifts to another attractor. We shall prove that, for some values of the parameters, these transition times happen with probability one and are unpredictable, in the sense that their distribution shows lack of memory. This phenomenon gives rise to a cycling behavior of the finite dimensional system: due to the sudden switching of the system towards the other attractor the trajectories oscillate for long times close to one of the two (metastable) equilibria. Put differently, it is as if there were two different time scales: on a short time scale, the equilibria described in Proposition 2 are, apparently, stable; on a longer time scale, we instead observe a cycling behavior generated by the endogenous fluctuations between the two regimes 18 , Let us focus on the large externality case and specifically on a situation in which multiple BGP equilibria exist (case ii.b in Proposition 2). In such a case two stable BGP equilibria exist; as discussed above which equilibrium our economy will achieve depends upon the initial conditions $\left(\chi_{0}, \varphi_{0}, x_{0}\right)$, and in particular a critical role is played by the initial condition on $x_{t}$. Indeed, the initial share of innovative firms determines whether the equilibrium share will be high or low, determining thus whether the economic growth rate, $\gamma$, will be high or low. While this outcome is clear in the infinitely large number of research firms version of the problem, whether this holds true also for the finite version is not so obvious. In fact, in the finite version of the model, research firms are subject to random shocks which determine whether they will decide to innovate or not; in the infinite version the effects of such shocks cannot be analyzed since the approximation provided by equation (18) turns out to be completely deterministic.

Let us denote by $x_{t}^{N}$ the proportion of innovative firms at time $t$ among a total population of $N$ firms. As said, under the assumptions of case ii.b in Proposition 2, the trajectory $x_{t}^{N}$ has a metastable behavior: it fluctuates close to one of the two equilibria, say $\bar{x}_{L}$ and, after a random time, it suddenly jumps to values close to $\bar{x}_{H}$ (and viceversa). We now state a proposition collecting the main properties of the so called tunneling time, i.e., the time needed for a trajectory to leave the basin of attraction of one equilibrium.

\footnotetext{
${ }^{17}$ When a unique equilibrium exists, the finite dimensional system is still stable in the sense that shocks generate fluctuations around the unique equilibrium. In this case, the long time scale effect is not present since there is no possibility of cycling between equilibria. We thus focus our discussion in this section on the most interesting case in which multiple equilibria exist and the implications of metastability.

${ }^{18}$ We would like to stress the fact that, as mentioned earlier, such a metastable behavior pertains also to the finite dimensional version of classical random utility models such as the Brock \& Durlauf (2001) model. To the best of our knowledge, such a peculiarity of this type of systems has never been discussed within the economics literature.
} 
The cycling behavior is due to a sequence of such (random) tunneling times. Unfortunately, the results describing the probabilistic properties of the tunneling times are rather technical; in order to make the discussion more clear, we prefer to maintain this section as simple as possible avoiding technicalities and postpone to Appendix $\mathrm{B}$ a more involved mathematical discussion. In order to define $T_{N}$, the tunneling time of the process $x_{t}^{N}$, we introduce two significant values in the state space of $x_{t}^{N}$ :

$$
x_{L}^{N}=\frac{\left\lceil N \bar{x}_{L}\right\rceil}{N} ; \quad x_{M}^{N}=\frac{\left\lceil N \bar{x}_{M}\right\rceil}{N} ;
$$

these are the values that the process $x_{t}^{N}$ can reach and that better approximate (from above) the real values $\bar{x}_{L}$ and $\bar{x}_{M}$, respectively. The tunneling time of the process $x_{t}^{N}$ is defined as the time needed to cross the basin of attraction of $\bar{x}_{H}$, when starting close to $\bar{x}_{L}$. More precisely,

$$
T_{N}=\min _{t>0}\left\{x_{t}^{N}=x_{M}^{N}, x_{0}^{N}=x_{L}^{N}\right\} .
$$

Note that this is exactly the first time at which the trajectory starting close to the equilibrium $\bar{x}_{L}$ will cross $\bar{x}_{M}$, hence entering the basin of attraction of $\bar{x}_{H}$. Once the process has crossed $\bar{x}_{M}$, it rapidly converges towards $\bar{x}_{H}$. The main properties of the tunneling time are summarized in the next proposition.

Proposition 3. Consider the process $x_{t}^{N}$ as described by (3) - (4). Assume that $J>J^{t}(\beta)$ and $h<h^{t}(J, \beta)$, where the thresholds are as defined in Proposition 2 . Then there exists a suitable constant $\Delta$ depending on $\beta, J, h$ such that the tunneling time $T_{N}$ has the following properties:

a) for all $\delta>0, \lim _{N \rightarrow \infty} \mathbb{P}\left(e^{N(\Delta-\delta)}<T_{N}<e^{N(\Delta+\delta)}\right)=1$;

b) $T_{N} / \mathbb{E}\left[T_{N}\right]$ converges in law to a unit-mean exponential random variable, as $N \rightarrow \infty$.

A formal proof of the proposition is presented in Appendix $\mathrm{B}$ where the explicit functional form of $\Delta$ is also provided. As suggested by Proposition 3, the random jumps happen with probability one for each trajectory, although the jump times $T_{N}$ could be possibly large. Specifically, the transition times tend, for $N \rightarrow \infty$, to an exponentially distributed random variable with expectation proportional to $e^{\Delta N}$, where $\Delta$ is a suitable constant depending on the parameters of the model. It turns out that, for values of $J$ close to $J^{t}$, the random time needed to exit the basin of attraction of the two locally stable equilibria is relatively small and trajectories showing growth cycles arise. In Figure 4 (right panel), we provide an example showing that, for $J=2.05$ (recall that $J^{t}=2$ ), this random time is reached early enough to be seen in the trajectory. More precisely, we show that the (stochastic) time series of $x_{t}^{N}$ may deviate from its expected behavior predicted by equation (18). Recall that $x_{t}$ describes exactly the deterministic evolution of the system under the modeling assumption that $N$ is infinite. In the left panel we show that $x_{t}^{N}$ may converge to the equilibrium it is not supposed to achieve. Indeed, since the initial condition $x_{0}=0.1$ is greatly lower than $\bar{x}_{M}=0.4178$, we would expect the time series of $x_{t}^{N}$ to fluctuate around the red-dashed trajectory $x_{t}^{(L)}$ leading to the low equilibrium. However, in this particular simulation, this is not the case: the trajectory deviates and start fluctuating around the high equilibrium $\bar{x}_{H}$. In the right panel, as said, we show that the finite dimensional trajectory $x_{t}^{N}$ may spend quite a long time close to one of the two equilibria and then depart from it to reach the other one. What discussed for $x_{t}^{N}$ has clear implications also in terms of the macroeconomic outcome: differently from what suggested by the (deterministic) theory, the system, even when the initial conditions are very close to the high BGP equilibrium, may converge towards the low BGP equilibrium or oscillate between the two BGP equilibria without converging to a steady state. Note that in the small externality case, in which the equilibrium is unique, such an effect naturally disappears. This suggests that government intervention may be essential not only to allow the economy to solve its eventual low growth trap problem but also to reduce the fluctuations (occurring with probability one) in economic activity.

We provide now some estimates of the expected tunneling time for the process $x_{t}^{N}$. When considering the values of the parameters as in Table 1, $h=0$ and $J=2.05$ (see Figure 4, right panel), $\Delta \approx 1.92 \cdot 10^{-4}$ 

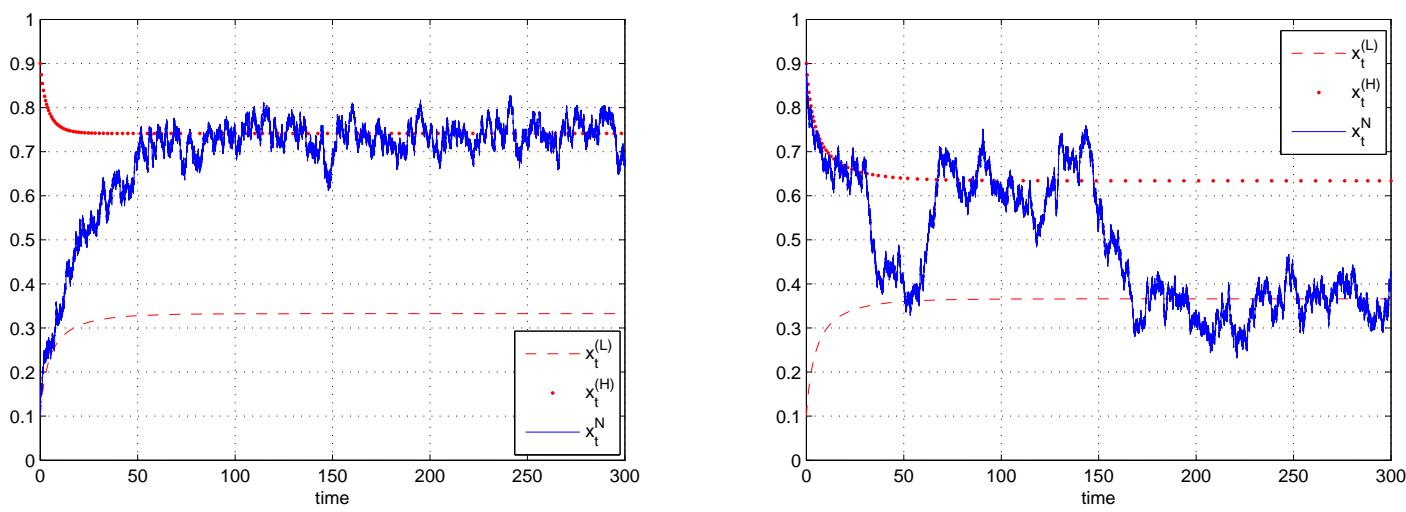

Figure 4: The evolution of $x_{t}^{N}$ (in blue) for a finite dimensional system of $N=1,000$ firms. In the left panel, we see the deviation from the expected trajectory $\bar{x}_{t}^{(L)}$ suggested by the model in favor of $\bar{x}_{t}^{(H)}$. In the right panel we have a trajectory fluctuating around the two attractors. Parameters are as in Table 1 with $h=0.01$ and $J=2.14$ (left panel) and $h=0$ and $J=2.05$ (right panel).

so that $e^{\Delta N} \approx 1.212$. This means that, on average, we expect $e^{\Delta N} \cdot N \approx 1212$ single transitions (i.e., single firms deciding to change their innovation policy) to actually observe a tunneling time. As a comparison, in Figure 5, we show a trajectory of $x_{t}^{N}$ where parameters are the same exept for $J$ which is now higher: $J=2.14$. In this case, $\Delta=3.217 \cdot 10^{-3}$ and $e^{\Delta N} \approx 24.965$. Now, we expect 24965 single transitions to observe a tunneling time. The comparison with the right panel of Figure 4 shows that, as expected, the tunneling times are now less frequent: a longer time scale is needed in order to capture the transitions from one BGP equilibrium to the other.

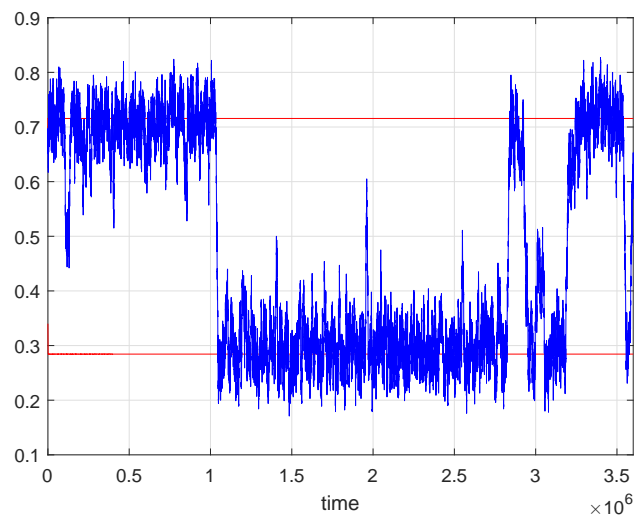

Figure 5: The evolution of $x_{t}^{N}$ (in blue) for a finite dimensional system of $N=1,000$ firms. Parameters are as in Table 1 with $h=0$ and $J=2.14$. The BGP innovation share equilibria are $\bar{x}_{H}=0.7156$ and $\bar{x}_{L}=0.2844$.

We conclude this section by providing an intuition about the effects of metastability on macroeconomic variables, and in particular on how metastibility results in output fluctuations generating thus a growth cycle. To this aim, in order to simplify computational problems we consider the special case in which the inverse of the intertemporal elasticity of substitution and the capital share perfectly coincide, that is $\sigma=\alpha$. Such a case has been frequently analyzed in order to fully characterize transitional dynamics in similar growth models (Smith (2006); Xie (1994)) since allowing to decouple some variables in the system (16) (18) and thus to obtain an explicit analytical expression for the evolution of main variables. Whenever 
$\sigma=\alpha$, it is straightforward to show that the evolution of capital is given by the following expression: $\dot{k}_{t}=\left(1-\frac{h}{\ell} x_{t}\right) A_{t} k_{t}^{\alpha}-\frac{\rho}{\alpha} k_{t}$, from which it is then possible to characterize the evolution of output from $y_{t}=A_{t} k_{t}^{\alpha}$. We wish to provide an intuition about the trajectories of $y_{t}^{N}$, the level of per capita output, under the assumption that $x_{t}$ is now substituted by $x_{t}^{N}$. This can be done by discretizing the system of differential equations to obtain a (approximated) difference equations system where the variables are now $\left(k_{t}^{N}, A_{t}^{N}, y_{t}^{N}\right)$ where $k_{0}^{N}=k_{0}, A_{0}^{N}=A_{0}$,

$$
\left\{\begin{array}{l}
k_{t+1}^{N}=k_{t}^{N}+\left(1-\frac{h}{\ell} x_{t}^{N}\right) A_{t}^{N}\left(k_{t}^{N}\right)^{\alpha}-\frac{\varphi}{\alpha} k_{t}^{N} \\
A_{t+1}^{N}=\phi A_{t}^{N} x_{t}^{N}
\end{array}\right.
$$

and $y_{t}^{N}=A_{t}^{N}\left(k_{t}^{N}\right)^{\alpha}$, with $y_{0}^{N}=A_{0}^{N}\left(k_{0}^{N}\right)^{\alpha}$. In Figure 6, we plot (on a log-scale) the trajectory of $y_{t}^{N}$ associated with the trajectory of $x_{t}^{N}$ presented in Figure 5 . It is straightforward to observe that the slope of $\log \left(y_{t}^{N}\right)$, representing the growth rate of per capita output, changes whenever a regime shift occurs: the slope is steeper when $x_{t}^{N}$ fluctuates around $\bar{x}_{H}$ while it is flatter when $x_{t}^{N}$ fluctuates around $\bar{x}_{L}$, meaning that the growth rate fluctuates between high and low values giving thus effectively rise to a growth cycle. Even if the difference in the growth rates might apparently seem small, this is not the case: from $\gamma=\frac{\phi \bar{x}}{1-\alpha}$, we can compute that the two BGP rates are substantially different; indeed, $\bar{\gamma}_{H}=0.0427$ and $\bar{\gamma}_{L}=0.0170$ suggesting a $2.5 \%$ difference in the growth rate between the high and low regime.
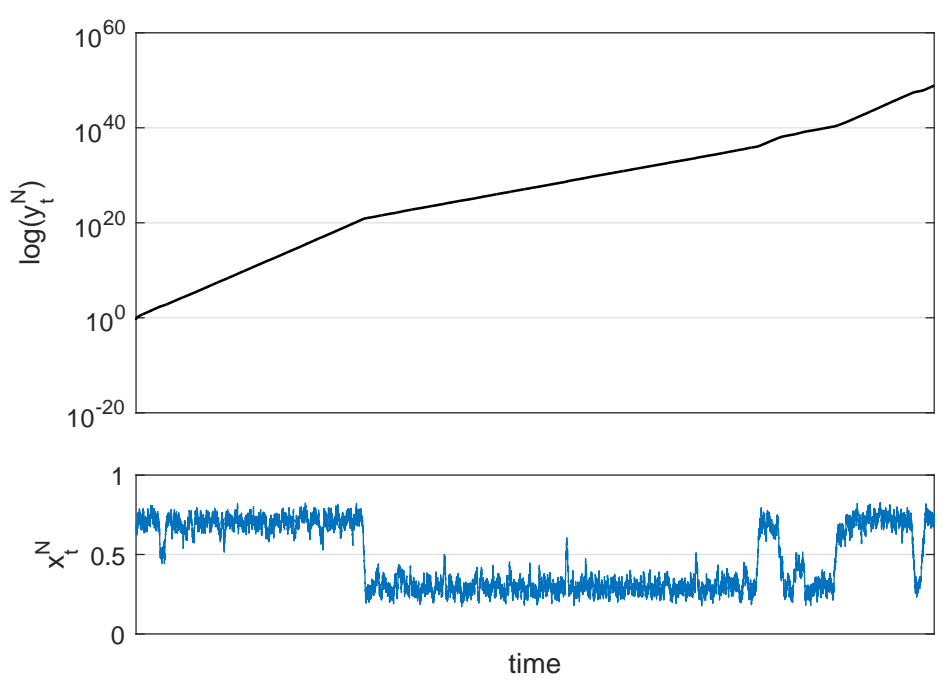

Figure 6: The evolution of $y_{t}^{N}$ on a logarithmic scale (top panel) associated with the evolution of $x_{t}^{N}$ (bottom panel).

\section{$6 \quad$ A Generalized Model}

The model that we have focused on thus far is based on the assumption that the incentive for innovative activities (i.e., the price paid by the government in order to purchase the innovation from research firms), $h$, is constant and exogenously given. This might seem a merely convenient ad hoc simplification allowing to decouple the research industry from other macroeconomic variables; however, as we shall see in a while even extending the analysis to a more general setting would lead to results qualitative similar to those just discussed in our baseline model. In order to look at this, we allow the incentive to be time-dependent and endogenous since depending on other macroeconomic variables. In this case the government budget constraint reads as follows:

$$
\tau_{t} \ell=h_{t} x_{t}
$$


where $h_{t}=H\left(x_{t}, A_{t}, t\right)$ for a suitable function $H: \mathbb{R}^{3} \rightarrow[0, \ell]$. Note that the upper bound is needed to ensure that $\tau_{t} \leq 1$ for all $t$. This specification of $h_{t}$ suggests that the government may wish to provide stronger or weaker incentives to innovative activities according to the overall level of technological advancement $A_{t}$ achieved in the economy. Therefore, the tax rate should also change with the level of technological advancement: the tax rate is now defined as $\tau_{t}=\mathcal{T}\left(A_{t}\right)$ where we do not impose any restriction a priori on the shape of the function $\mathcal{T}(\cdot)$. The above budget constraint in this case read as follows:

$$
h_{t}=H\left(x_{t}, A_{t}, t\right)=\frac{\ell}{x_{t}} \mathcal{T}\left(A_{t}\right)
$$

We will discuss one specific example at the end of this section, but we firstly provide a generalization of Proposition 1 and Proposition 2. Note that, since now $h_{t}=H\left(x_{t}, A_{t}, t\right)$, the Markov process $x_{t}^{N}$ and the relative limit $x_{t}$ are no longer disentangled from other macroeconomic variables: the dependence in $A_{t}$ makes the derivation of the law of large numbers more complicated 19 . Indeed, we need to define a suitable twodimensional stochastic process $\left(x_{t}^{N}, A_{t}^{N}\right)$ such that, when taking the limit for $N \rightarrow \infty,\left(x_{t}^{N}, A_{t}^{N}\right)$ converges to the pair $\left(x_{t}, A_{t}\right)$ defined by $(14)-(15)$. This is formally stated in the following proposition.

Proposition 4. For all $N \geq 1$, consider the stochastic process $\left(x_{t}^{N}, A_{t}^{N}\right)$ defined as:

$$
x_{t}^{N}=\frac{1}{N} \sum_{i=1}^{N} \omega_{i, t}, \quad A_{t}^{N}:=A_{0}^{N} e^{\int_{0}^{t} \phi x_{s}^{N} d s}
$$

where $\phi>0$ and

$$
\mathbb{P}\left(\omega_{i, t+\Delta t}=1 \mid \omega_{i, t}, x_{t}^{N}, A_{t}^{N}\right)=\eta\left[H\left(x_{t}^{N}, A_{t}^{N}, t\right)-z+J\left(x_{t}^{N}-\frac{1}{2}\right)\right] .
$$

Moreover, assume that $\lim _{N \rightarrow \infty} x_{0}^{N}=x_{0}$ and $\lim _{N \rightarrow \infty} A_{0}^{N}=A_{0}$. Then, when $N \rightarrow \infty$, the process $\left(x_{t}^{N}, A_{t}^{N}\right)$ weakly converges to $\left(x_{t}, A_{t}\right)$ solving

$$
\begin{aligned}
\dot{x}_{t} & =\frac{1}{2} \tanh \left\{\beta\left[H\left(x_{t}, A_{t}, t\right)+J\left(x_{t}-\frac{1}{2}\right)\right]\right\}-x_{t}+\frac{1}{2} \\
\dot{A}_{t} & =\phi x_{t} A_{t}
\end{aligned}
$$

with initial conditions $\left(x_{0}, A_{0}\right)$.

Proof. Note that, in principle, the definition of $A_{t}^{N}$ introduces a dependence on the past of the process $x_{t}^{N}$, thus a loss of Markovianity. Nevertheless, we will see that the pair $\left(x_{t}^{N}, A_{t}^{N}\right)$ is still Markovian. To this aim, we introduce the infinitesimal generator of the process $\left(x_{t}^{N}, A_{t}^{N}\right)$ applied to functions $f: \mathbb{R}^{3} \rightarrow \mathbb{R}$ with compact support:

$$
\begin{aligned}
\mathcal{L}_{N} f(x, A, t)= & N x_{t}^{N} \eta^{-}\left(x_{t}^{N}, A_{t}^{N}\right)\left[f\left(x-\frac{1}{N}, A, t\right)-f(x, A, t)\right]+ \\
& N\left(1-x_{t}^{N}\right) \eta^{+}\left(x_{t}^{N}, A_{t}^{N}\right)\left[f\left(x+\frac{1}{N}, A, t\right)-f(x, A, t)\right]+ \\
& \phi x_{t}^{N} A_{t}^{N} f_{A}^{\prime}(x, A, t)+f_{t}^{\prime}(x, A, t)
\end{aligned}
$$

where

$$
\eta^{+}(x, A):=\eta\left(H(x, A, t)-z+J\left(x-\frac{1}{2}\right)\right)
$$

\footnotetext{
${ }^{19}$ We are indebted to an anonymous referee for suggesting such a non-trivial model's extension. Apart from generalizing our previous results, this allows us to discuss the mathematics behind this formulation with endogenous $h_{t}=H(\cdot)$ and compare it with the classical random utility model with constant $h$. To the best of our knowledge, such a type of generalization has never been discussed in the literature thus far, not even in other frameworks.
} 
represents the probability for an agent with $\omega_{i, t}=0$ to become active (i.e., to decide to do research) at time $t$ and $\eta^{-}=1-\eta^{+}$. Since $\mathcal{L}_{N}$ only depends on the variables $\left(x_{t}^{N}, A_{t}^{N}\right)$ computed at time $t$, we conclude that the stochastic process $\left(x^{N}, A^{N}\right)$ is Markovian.

Moreover, since all the derivatives are uniformly bounded, it turns out that

$$
\lim _{N \rightarrow \infty} \sup _{(x, A, t) \in \mathbb{R}^{3}}\left|\mathcal{L}_{N} f(x, A, t)-\mathcal{L} f(x, A, t)\right|=0
$$

where

$$
\mathcal{L} f(x, A, t)=\left[-x+\eta^{+}\right] f_{x}^{\prime}(x, A, t)+\dot{A} f_{A}^{\prime}(x, A, t)+f_{t}^{\prime}(x, A, t) .
$$

The form of $\mathcal{L}$ provided in $(30)$ follows from the fact that, taking the first order approximation of $\mathcal{L}_{N}$,

$$
\begin{aligned}
\mathcal{L}_{N} f(x, A, t)= & N x_{t}^{N} \eta^{-}\left(x_{t}^{N}, A_{t}^{N}\right)\left(-\frac{1}{N}\right) f_{x}^{\prime}(x, A, t)+ \\
& N\left(1-x_{t}^{N}\right) \eta^{+}\left(x_{t}^{N}, A_{t}^{N}\right)\left(\frac{1}{N}\right) f_{x}^{\prime}(x, A, t)+ \\
& \phi x_{t}^{N} A_{t}^{N} f_{A}^{\prime}(x, A, t)+f_{t}^{\prime}(x, A, t)+o\left(\frac{1}{N}\right) ;
\end{aligned}
$$

which can be rewritten as

$$
\begin{aligned}
& \mathcal{L}_{N} f(x, A, t)=\left[-x_{t}^{N}+\eta^{+}\left(x_{t}^{N}, A_{t}^{N}\right)\right] f_{x}^{\prime}(x, A, t)+ \\
& \quad \phi x_{t}^{N} A_{t}^{N} f_{A}^{\prime}(x, A, t)+f_{t}^{\prime}(x, A, t)+o\left(\frac{1}{N}\right) .
\end{aligned}
$$

Now it is easy to show that the limit of $\mathcal{L}_{N}$ is $\mathcal{L}$ as expressed in 30. . Note that $\mathcal{L}$ is the infinitesimal generator of the process $\left(x_{t}, A_{t}\right)$, where

$$
\dot{x}_{t}=-x_{t}+\eta^{+}\left(x_{t}, A_{t}\right) ; \quad \dot{A}_{t}=A_{t} \phi x_{t} .
$$

This can be seen computing $\mathcal{L} x$ and $\mathcal{L} A$ (i.e., considering $f \equiv x$ and $f \equiv A$, respectively). Finally, by the functional form of $\eta^{+}$, we easily see that

$$
\dot{x}_{t}=-x_{t}+\frac{1}{2}+\frac{1}{2} \tanh \left(H\left(x_{t}, A_{t}, t\right)-z+J\left(x_{t}-\frac{1}{2}\right)\right) .
$$

By virtue of Theorem 1.6.1 in Ethier and Kurtz (1986), equation $(29)$ and the assumption on the convergence of the initial conditions ensure that the stochastic process $\left(x_{t}^{N}, A_{t}^{N}\right)$ weakly converges to $\left(x_{t}, A_{t}\right)$.

Apart from the complication introduced by the need to deal with a two-dimensional stochastic process, the results are qualitatively identical to those discussed earlier. When the number of research firms is infinitely large, we can approximate the model's outcome through some deterministic differential equations describing the evolution of the innovation share and the evolution of technology. Differently from what seen earlier in our baseline setup, now the research industry and the macroeconomic outcome are more realistically mutually interconnected. By having generalized Proposition 1, it is now straightforward to derive a generalization of Proposition 2 .

Proposition 5. Consider the economy described by equations (16) - 18 where $h_{t}=H\left(x_{t}, A_{t}, t\right)$ for a differentiable function $H: \mathbb{R}^{3} \rightarrow[0, \ell]$ such that $\lim _{t \rightarrow \infty} h_{t}=\bar{h} \in[0, \ell]$ is well defined. Then, along the (asymptotic) BGP equilibria the economic growth rate is given by:

$$
\gamma=\frac{\phi \bar{x}}{1-\alpha}
$$

where $\bar{x}$ is the solution of:

$$
\bar{x}=\frac{1}{2} \tanh \left\{\beta\left[\bar{h}+J\left(\bar{x}-\frac{1}{2}\right)\right]\right\}+\frac{1}{2} .
$$


Proof. Just take the limit for $t \rightarrow \infty$ and impose $\dot{x}=0$ in (32). The rest of the proof follows arguing similary as in Proposition 2,

Proposition 5 characterizes the BGP in our generalized model in which the innovation incentive is endogenous and time-varying. Note that the economic growth rate is the same as in our baseline model and the only eventual difference between the two frameworks is due to the eventual different equilibrium share of innovative firms. Concerning multiplicity/uniqueness of equilibria, the whole discussion of Proposition 2 still holds true as long as $h$ is replaced by $\bar{h} \in[0, \ell]$. Indeed, two thresholds $J^{t}(\beta)$ and $h^{t}(J, \beta)$ still exist although, differently from the baseline case, we are not able to characterize them explicitly. Propositions 4 and 5 jointly suggest that the results previously discussed in our baseline setup still hold true even in a more sophisticated model in which innovation decisions are endogenous and related to macroeconomic outcomes. Therefore, also the related discussion of policy implications still apply, confirming the importance of taking into account social interaction within the research industry in order to understand the determinants of macroeconomic performance. Finally, also in this more general case, when multiple BGP equilibria exist, they are metastable in the sense of what discussed in Section 5 , therefore, the trajectories of $x_{t}^{N}$ will exhibit cycling patterns and endogenous fluctuations will occur over the long run.

We exemplify the above discussion by considering some specific functional form for $h_{t}$ in order to further clarify the results.

Example 6.1. Let us consider: $h_{t}=H\left(x_{t}, A_{t}, t\right)=\frac{\ell}{x_{t}} \mathcal{T}\left(A_{t}\right)$, where $\mathcal{T}\left(A_{t}\right)=\tau \cdot\left(1+e^{-A_{t}}\right)$, for $0<\tau \leq 1 / 2$. In this case, $\bar{h}=\frac{\tau \ell}{\bar{x}}$. In Figure 7 , we plot the value of the BGP growth rate $\gamma$ as a function of the main parameter. In the left panel we set $\tau=4 \cdot 10^{-5}$ and we let $J$ vary, while in the right panel we set $J=3$ and we let $\tau$ vary; all other parameter values are set accordingly to Table 1 . We can see that the bifurcation diagram is similar to what described in our baseline model. Finally, note that in case of multiplicity of equilibria, the equilibrium value for the tax rate and the innovation incentive $\bar{h}$, change with the equilibrium prevailing in the economy. For instance, for $J=3$ and $\tau=4 \cdot 10^{-5}, \bar{h}$ takes value 0.2060 or 0.0427 if $x_{L}$ or $x_{H}$, respectively.
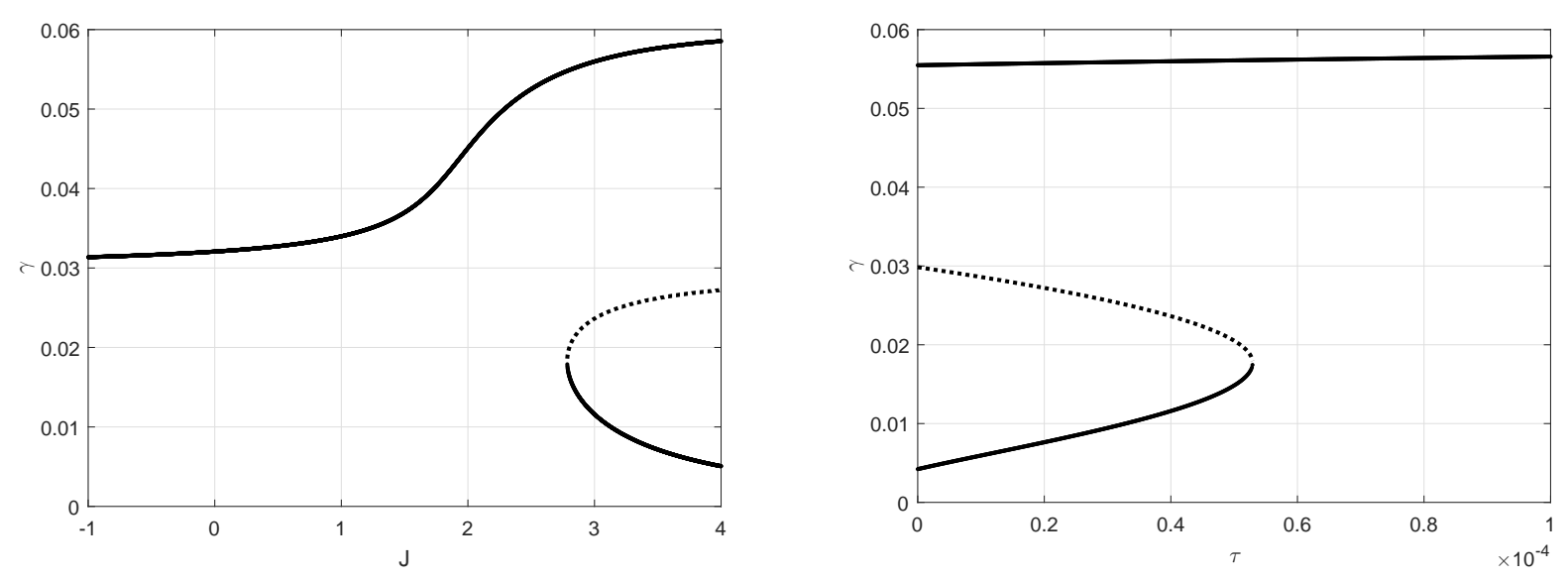

Figure 7: Changes in the growth rate, $\gamma$, for different values of $J$ (left panel) with $-1 \leq J \leq 4$ and $h$ (right panel) with $0 \leq h \leq 0.0001 ; \tau=4 \cdot 10^{-5}$ in the left panel and $J=3$ in the right panel.

Example 6.2. Consider the case of $\tilde{h}_{t}=h_{t} \cdot y_{t}$, where $h_{t}=H\left(x_{t}, A_{t}, t\right) \in[0, \ell]$ and $y_{t}$ is the time-varying per capita output. In this case, 26) reads as follows:

$$
\mathbb{P}\left(\omega_{i, t+\Delta t}=1 \mid \omega_{i, t}, x_{t}^{N}, A_{t}^{N}\right)=\eta\left[H\left(x_{t}^{N}, A_{t}^{N}, t\right) \cdot y_{t}-z+J\left(x_{t}^{N}-\frac{1}{2}\right)\right] .
$$


It is not difficult to show that Propositions 4 and 5 still apply. The result is however different: being $H$ bounded and $y_{t}$ diverging to infinity when $t \rightarrow \infty$, in (27) we have that $\bar{h}$ is replaced by $\lim _{t \rightarrow+\infty} \tilde{h}_{t}=+\infty$. Therefore, in this case, the unique solution to (27) is now $\bar{x}=1$. This is rather obvious: if the incentive to innovation explodes, it is worth to enter the market of innovation and the result is trivial.

\section{$7 \quad$ Middle-Income Trap}

An interesting line of interpretation of our stylized model is related to the middle-income trap hypothesis 20 , This refers to the experience common to many developing countries (especially in Latin America and in the Middle East) in the second half of the XIX century, in which growth has significantly slowed down after a first stage of take off characterized by rapid growth (see Gill and Kharas (2007), Commission on Growth and Development (2008)). This development process has allowed these economies to quickly move from a low-income to a middle-income status, but not to make the further leap needed to become high-income economies. This has advanced the hypothesis that there may exist a middle-income trap, preventing thus some economies to fill the gap with more advanced countries. What might be the specific hindrances affecting this second stage of economic development is still an open question, but these are likely to be substantially different from those involving the first stage in which traditional poverty traps are in place.

Understanding what may be the reason why some fast growing economies have failed to achieve a highincome status is an active and recent research question with clear policy implications. While empirical evidence supporting the existence of a middle-income trap seems robust and convincing, much less clear is from a theoretical point of view why fast growth might come to an end. On the empirical side, Eichengreen et al. (2012) show that growth tends to slowdown at levels of per capita income of about $\$ 15,000$ (at 2005 constant international PPP prices), suggesting that a critical role is played by a reduction in the growth rate of the total factor productivity (TFP); specifically, a drop in TFP growth represents about $85 \%$ of the fall in per capita income growth. Eichengreen et al. (2013) provide some additional evidence, showing that the distribution of growth slowdowns is not necessarily unimodal, and in particular two modes, one around $\$ 15,000$ and another around $\$ 11,000$, exist. On the theoretical side, very few works have tried to provide some explanation of growth slowdowns in middle-income countries, and they focus on reallocation or misallocation of workers between different economic sectors. A traditional argument suggests that while in earlier stages of development it may be possible to raise productivity by shifting workers from agriculture to industry, this process may come to an end whenever the share of workers employed in agriculture falls enough (Lewis (1954)). A more recent explanation emphasizes that a low allocation of high skilled individuals in the research sector may give rise to low productivity growth; however, this situation of potential slow growth can be fixed by policy interventions (Agénor and Canuto (2015)). Differently from these works in which intersector dynamics is the driver of eventual growth slowdowns, our paper provides an alternative explanation based entirely on social interactions and technology diffusion.

Along the lines of Agénor and Canuto (2015), whenever the economy experiences multiple BGP equilibria (Proposition 2, case ii.b), the intermediate BGP equilibrium $\gamma_{M}$ (i.e., the low growth trap threshold) can be clearly interpreted as a middle-income trap, separating fast and slow growing economies. Note that the eventual existence of such a trap is determined by the outcome in the research industry, which is completely driven by social interactions among research firms. Thus, the research intra-sector dynamics only might explain why technological progress and thus economic growth tend to be high or low in specific economies. In order to relate this to the pattern advanced by the middle-income trap hypothesis we need to understand why an economy initially (during a first stage of economic development) in a BGP with high economic growth rate, $\gamma_{H}$, may end up (in the second stage of development) in a BGP equilibrium with low growth rate, $\gamma_{L}$, later. In our setting this is equivalent to a either a fall in the number of innovative firms $x_{t}$ or a

\footnotetext{
${ }^{20}$ The term "middle-income trap" has been originally introduced by Gill and Kharas (2007), and the notion has also often been referred to as "growth slowdown" (Eichengreen et al. (2012)).
} 
rise in the intermediate equilibrium of the innovation share, $\bar{x}_{M}$; both the cases imply that an economy with an original high innovation share may end up with a low (compared with the critical threshold) innovation share and thus experiencing a slowdown in its economic growth. The former case may be triggered by a change in international policy, and specifically it may occur as a result of the introduction of tariffs or other restrictive policies, which by providing negative incentives for foreign firms to operate on the domestic market, leads some foreign firms engaged in research activity to exit the domestic research market. The latter case may instead be triggered by a change in domestic economic policy, and it may occur as a result of a reduction in the support provided to research firms which, by determining the amount of revenue received by innovative firms, tends to increase the intermediate equilibrium value of the share of firms engaged in research activities. While empirical evidence seems to supports our conclusions related to the negative relation between growth slowdowns and openness (Eichengreen et al. (2012)), the available evidence does not allow to either support or refute those related to the positive (up to a certain point) nexus between growth slowdowns and research-enhancing policies.

Apart from the eventual existence of such a middle-income trap, our model differently from Agénor and Canuto (2015) suggests that also growth cycles may occur. This implies that also fast growing economies cannot claim to have definitely escaped their middle-income trap, since they may be cyclically pulled into situations of growth slowdowns. This reinforces our previous conclusions that policymakers can play a critical role in the development process. By actively intervening with specific policies they can completely solve the trap problem dampening the size of the growth fluctuations, promoting a smooth process of fast growth allowing the economy to eventually catch up with more advanced economies and become a high-income country.

\section{Conclusion}

Technological progress is by far the most important determinant of economic growth over the long run. However, whether and how the interaction among research firms in the research industry might determine technological progress has never been analyzed thus far in the growth literature. Thus, in this paper we have tried to fill this gap by allowing a certain degree of firms interaction. Specifically, we assume that firms decide whether to innovate or not by taking into account also what other research firms do. Such an interaction among research firms, by determining the rate of technological progress, plays a critical role in shaping the whole macroeconomic outcome. Indeed, we have shown that under certain parameter conditions, by mimicking the behavior of the share of innovative firms, the economy may be characterized by a multiplicity of BGP equilibria and eventually may face a situation of low growth trap. We have also shown that the economy may eventually (endogenously) fluctuate between the low and high BGP generating thus a growth cycle in which periods of low and high economic growth rates follow one another. The potential existence of low growth traps and endogenous growth cycles suggest that the government might play an essential role in order to contrast such negative effects. In particular, by rising enough the tax rate applied to households' income it could completely solve the low growth trap problem, avoiding thus further fluctuations in economic activity. All these results are robust in the sense that they hold true both in our baseline model in which the innovation incentive is constant and in its generalized version in which this is potentially time-varying and dependent upon other macroeconomic variables.

This paper represents a first attempt to enrich the macroeconomic dynamics in traditional models of endogenous growth by allowing a certain extent of externality in research decisions. The approach followed is thus quite simplistic on purpose in order to show in the simplest possible way (which is already all but simple from a mathematical point of view) which might be the potential implications of allowing for social interactions in traditional macroeconomic models. Of course, our framework has several limitations which need to be accounted for in future research. Specifically, the dichotomous choice of research firms to do or not to do research does not allow to quantify research efforts; this assumption needs to be relaxed in order 
to fully characterize research decisions and outcomes. Also the specification of the research market is overly simple, and adopting a more traditional setup with either horizontal or vertical product differentiation may shed some further light on the impacts of social interactions on macroeconomic outcomes. Extending the analysis along these lines is left for future research.

\section{A The Rationale behind Random Utility Models}

In this appendix we briefly summarize the main ideas recovered by Brock \& Durlauf (2001) and leading to the profit structure defined in (1). Suppose that a research firm faces the binary decision to innovate or not to innovate. We define the binary random variable $\omega \in\{0,1\}$ accordingly. The main assumption behind random utility models is that the profit $\pi$ related to the innovation has the following general structure:

$$
\pi\left(\omega_{i}\right)=R\left(\omega_{i}, \mu_{i}^{e}\left(\omega_{-i}\right), h\right)-\zeta\left(\omega_{i}\right),
$$

where revenues $R$ depend on the choice made by the firm, on the price $h$ received by the buyer of the innovation and by an externality term. Indeed, each firm $i$ estimates the conditional probability measure $\mu_{i}^{e}$ on the choices of others, where $\omega_{-i}$ denotes the vector of actions deprived of the $i$-th component. As seen in Section 2, costs are random and denoted by $\zeta$. For the moment, we set $z=0$ for simplicity.

We now make some further (minimal) assumptions to came up with a tractable profit structure.

i) $\pi(0)=0$. This is an obvious normalization. Both $R$ and $\zeta$ are zero if no research activity is in place. Therefore, we concentrate on $\pi(1)$ (we call it simpy $\pi$ ). Rearranging variables and notations we have:

$$
\pi=R\left(\mu_{i}^{e}\left(\omega_{-i}\right), h\right)-\zeta_{i} .
$$

ii) Externalities due to the behavior of competitors, only depend on the average action of others' choice. This implies that $\mu_{i}^{e}\left(\omega_{-i}\right)$ is substituted by the (simpler) statistics $x_{i}^{e}=\frac{1}{N-1} \sum_{j \neq i} x_{i j}^{e}$, where $x_{i j}^{e}=$ $\mathbb{E}^{(i)}\left[\omega_{j}\right]$ denotes the expectation of firm $i$ about the choice of competitor $j$. Therefore,

$$
\pi=R\left(x_{i}^{e}, h\right)-\zeta_{i} .
$$

Concerning the information structure of the model, we also assume that $\mathbb{E}^{(i)}[\cdot]=\mathbb{E}^{(j)}[\cdot]$ for all $i, j=$ $1, \ldots, N$. This amounts in saying that all firms share the same expectations about others' choices.

iii) We assume that $\frac{\partial \pi}{\partial x_{i}^{e}}=J$. This simplifying assumption introduces a unique parameter $J$ measuring the degree of dependence (or the force of externality) due to the others' actions. Note that $J>0$ resembles a staying-on-the-shoulder situation, whereas $J<0$ a fishing-out case. Secondly, as obvious, $\frac{\partial \pi}{\partial h}>0$.

iv) We assume that the pecuniary effects due to the sale of the technology and the externalities are additive. Moreover, for sake of simplicity, we assume a linear dependence. This fact, together with assumption iii), produces the following payoff:

$$
\pi=h+J x_{i}^{e}-\zeta_{i}
$$

v) Finally, we slightly correct $x_{i}^{e}$ by substituting it with $x_{i}^{e}-\frac{1}{2}$. The reason is that we want the decision to be driven by what the majority of the population of firms is doing. The quantity $x_{i}^{e}-\frac{1}{2}$ reflects exactly this goal: it is positive if and only if the majority of the research firms produces an innovation. Therefore, in case of a positive $J$, the single firm is more prone to align with the majority. On the contrary, if $J<0$, the firm will tend to behave in the opposite direction. We obtain:

$$
\pi=h-\zeta_{i}+J\left(x_{i}^{e}-\frac{1}{2}\right) .
$$


Therefore, by reintroducing a private cost $z$ and recalling that $\pi(0)=0$, we obtain the general expression for $\pi$ as it appears in (1):

$$
\pi\left(\omega_{i}\right)=\omega_{i}\left[h-\left(z+\zeta_{i}\right)+J\left(x_{i}^{e}-\frac{1}{2}\right)\right] .
$$

By applying the payoff structure defined above, we can verify that

$$
\omega_{i}=1 \Longleftrightarrow \pi(1) \geq \pi(0) \Longleftrightarrow h-\left(z+\zeta_{i}\right)+J\left(x_{i}^{e}-\frac{1}{2}\right) \geq 0 .
$$

The probabilistic structure of the model implies that for all $i=1, \ldots, N$,

$$
\mathbb{P}\left(\omega_{i}=1\right)=\mathbb{P}\left(h-\left(z+\zeta_{i}\right)+J\left(x_{i}^{e}-\frac{1}{2}\right) \geq 0\right)=\mathbb{P}\left(\zeta_{i} \leq h-z+J\left(x_{i}^{e}-\frac{1}{2}\right)\right) .
$$

Since agents receive different private signals, agents may have a different feeling about the best choice. Heterogeneity gives rise to the non-trivial equilibria and the (possible) multiplicity discussed in Proposition 2. In the case of a completely deterministic model (i.e., $\zeta_{i}=0$ for all $i$ ), agents would be homogeneous and we would obtain:

$$
\mathbb{P}\left(0 \leq h-z+J\left(x_{i}^{e}-\frac{1}{2}\right)\right) \in\{0 ; 1\},
$$

meaning that either $\omega_{i}=0$ or $\omega_{i}=1$ for all $i=1, \ldots, N$. The same reasoning extends to the continuous-time counterpart described by (4): assuming no randomness, there would be no space for any dynamics, and the outcome would be to a large extent trivial with all firms deciding either to innovate or not to innovate. In the body of the paper we thus focus on the most interesting situation in which agent heterogeneity gives rises to nontrivial dynamics. Most of our qualitative results in Proposition 2 would still hold true in the absence of heterogeneity case, but in this case the BGP equilibrium would be necessarily unique and characterized by either one of the two extreme long run growth rates $\gamma=\frac{\phi}{1-\alpha}$ if $\bar{x}=1$ or $\gamma=0$ if $\bar{x}=0$.

\section{B Proof of Proposition 3}

The proof of Proposition 3 basically follows Theorem 4.6 in Olivieri and Vares (2005). In order to make this reading as much self consistent as possible, we sketch the proof rearranged to match our model and our notations. We firstly specify the functional form for $\Delta$. To this aim, we introduce the so called "Gibbs free energy" 21.

$$
f_{\beta, J, h}(m)=-\left(\frac{J}{4} m^{2}+h m\right)+\frac{1}{\beta} \cdot \varepsilon(m),
$$

where

$$
\varepsilon(m)=\frac{1+m}{2} \ln \left(\frac{1+m}{2}\right)+\frac{1-m}{2} \ln \left(\frac{1-m}{2}\right) .
$$

Finally, define

$$
\Delta=\beta\left(f\left(m_{M}\right)-f\left(m_{L}\right)\right)
$$

where $f$ is as defined in (35), $m_{M}=2 \bar{x}_{M}-1$ and $m_{L}=2 \bar{x}_{L}-1$ and where $\bar{x}_{L}$ and $\bar{x}_{M}$ are, respectively, the smallest and the middle solutions (recall that, under our assumptions on the values of the parameters, this equation admits three real solutions $\left.\bar{x}_{L}<\bar{x}_{M}<\bar{x}_{H}\right)$ to

$$
\frac{1}{2} \tanh \left\{\beta\left[h-z+J\left(x_{t}-\frac{1}{2}\right)\right]\right\}-x_{t}+\frac{1}{2}=0 .
$$

\footnotetext{
${ }^{21}$ In statistical mechanics, the Gibbs free energy characterizes the potential associated with the states of the system. In particular, it can be proved that the equilibria $m_{L}$ and $m_{H}$ are local minimum points for $f$, whereas $m_{M}$ is a local maximum point.
} 
In what follows, we organize the proof Proposition 3 into four steps. In the first step we provide a lower bound for $T_{N}$, in the second an upper bound. Finally, we prove part (a) and part (b) of the proposition. As said, we only sketch the main results and refer the reader to Olivieri and Vares (2005) for further details. Our aim is mainly to let the reader appreciate the probabilistic properties, which this proposition relies on.

i) There exists a positive constant $c_{1}$ such that, for $N$ large enough, and each positive integer $T$,

$$
\mathbb{P}\left(T_{N} \leq T\right) \leq c_{1} T e^{-N \Delta} .
$$

This fact follows from the properties of the stationary distribution of a Markov chain. Indeed, let us define the stationary measure of $\left(x_{t}^{N}\right)_{t \geq 0}$ as $\nu_{N}$. It can be proved that

$$
\mathbb{P}\left(T_{N} \leq T\right) \leq T \cdot \frac{\nu_{N}\left(x_{M}^{N}\right)}{\nu_{N}\left(x_{L}^{N}\right)}=T \cdot e^{-N \beta\left(f\left(m_{M}\right)-f\left(m_{L}\right)\right)} .
$$

On the other hand, for $N$ large enough, $\beta f\left(m_{M}\right)-f\left(m_{L}\right) \geq \Delta-\frac{c_{2}}{N}$ for a suitable constant $c_{2}$. Therefore, (36) easily follows by putting $c_{1}=e^{c_{2}}$.

ii) For any positive sequence $\left(\varphi_{N}\right)_{N \geq 1}$ such that $\varphi_{N} \rightarrow \infty$,

$$
\mathbb{P}\left(T_{N} \geq e^{N \Delta} N \varphi_{N}\right)=0 .
$$

This follows from the fact that, for suitable constants $c_{3}$ and $c_{4}$,

$$
c_{3} e^{N \Delta} \leq \mathbb{E}\left(T_{N}\right) \leq c_{4} N^{2} e^{N \Delta} .
$$

For details on the proof of (38), we refer to Corollary 4.9 in Olivieri and Vares (2005). From (38) and applying the Markov inequality, we obtain (37).

iii) Point (a) of Proposition 3 follows from the fact that

$$
1-\mathbb{P}\left(\frac{1}{\varphi_{N}} e^{N \Delta}<T_{N}<e^{N \Delta} N \varphi_{N}\right)=\mathbb{P}\left(T_{N} \leq \frac{1}{\varphi_{N}} e^{N \Delta}\right)+\mathbb{P}\left(T_{N} \geq e^{N \Delta} N \varphi_{N}\right) .
$$

Both terms of the RHS go to zero for any positive sequence $\left(\varphi_{N}\right)_{N \geq 1}$ such that $\varphi_{N} \rightarrow \infty$ due to $i$ ) and $i$ ), respectively. This proves part (a) in Proposition 3 .

iv) Define the sequence of random variables $\left(\tilde{T}_{N}\right)_{N \geq 2}$, where $\tilde{T}_{N}:=T_{N} / \gamma_{N}$ and where $\gamma_{N}$ is such that

$$
\lim _{N \rightarrow \infty} N^{-1} \ln \left(\gamma_{N}\right)=\Delta
$$

It can be shown that this sequence is tight and its limits $\tau$ along subsequences have the property that

$$
\mathbb{P}(\tau>t+s)=\mathbb{P}(\tau>t) \mathbb{P}(\tau>s) .
$$

This, in turns, shows that $\tilde{T}_{N}$ is asymptotically exponential, thus, memoryless. Finally,

$$
\lim _{N \rightarrow \infty} \mathbb{E}\left[\tilde{T}_{N}\right]=\int_{0}^{+\infty} \lim _{N \rightarrow \infty} \mathbb{P}\left(T_{N}>s \gamma_{N}\right) d s=\int_{0}^{+\infty} e^{-s} d s=1,
$$

and this concludes the proof of Proposition 3 .

To be precise, what we have shown in point $i v$ ) is true for a stopped version of $x^{N}$ : consider $\tilde{x}^{N}$ where $\tilde{x}_{t}^{N}$ has the same transition probabilities of $x_{t}^{N}$ for $t \leq T_{N}$ and $\tilde{x}_{t}^{N} \equiv \tilde{x}_{T_{N}}^{N}$ for $t \geq T_{N}$ (it is a stopped version of the original process at time $T_{N}$ ). It can be proved that the two processes are coupled up to $T_{N}$, so that the their probabilistic features are the same. Since we are interested in the trajectories up to $T_{N}$, working with $x^{N}$ or $\tilde{x}^{N}$ is exactly the same to our purposes. 
Note, finally, that the transition from $\bar{x}_{L}$ to $\bar{x}_{H}$ can be analyzed exactly in the same way, by simply considering $\Delta=\beta\left(f\left(m_{M}\right)-f\left(m_{H}\right)\right)$. We would like to stress the fact that, differently from the common notion of cycles in macroeconomics in which their periodicity is highly irregular and stochastic, in probability theory the notion of cycles requires the periods of the transitions to be deterministic and constant. According to this latter view, the tunneling time $T_{N}$ should converge to 1 , rather that to an exponential random time with average 1 as stated in Proposition 3. Therefore, even if this is not totally correct from a probabilistic point of view, in our discussion we adopt the macroeconomic view and terminology by referring to the metastability property as a cycling behavior.

\section{References}

Agénor, P.R., Canuto, O. (2015). Middle-income growth traps, Research in Economics 69, 641-660

Azariadis C., Stachurski J. (2005). Poverty traps, in (Aghion P., Durlauf S., Eds.) "Handbook of Economic Growth", vol. 1, ch. 5 (Elsevier)

Acemoglu, D. (2009). Introduction to modern economic growth (Princeton, NJ: Princeton University Press)

Aghion, P., Howitt, P. (1992). A model of growth through creative destruction, Econometrica 60, 323-351

Anderson, S., De Palma, A., Thisse, J.-F. (1992). Discrete choice theory of product differentiation (Cambridge: MIT Press)

Bambi, M., Gozzi, F., Licandro, O. (2014). Endogenous growth and wave-like business fluctuations, Journal of Economic Theory 154, 68-111

Barro R.J., Sala-i-Martin X. (1995). Economic Growth (MIT Press: Cambridge)

Bass, F.M. (1969). A new product growth for model consumer durables, Management Science 15, 215-227

Barucci, E., Tolotti, M. (2012). Social interaction and conformism in a random utility model, Journal of Economics Dynamics \& Control 36, 1855-1866

Benhabib, J., Farmer, R.(1994). Indeterminacy and increasing returns, Journal of Economic Theory 63, $19-41$

Benhabib, J., Nishimura, K. (1998). Indeterminacy and sunspots with constant returns, Journal of Economic Theory, 81, 58-96

Blume, L., Durlauf, S. (2003). Equilibrium concepts for social interaction models, International Game Theory Review 5, 193-209

Brock, W., Durlauf, S. (2001). Discrete choice with social interactions, Review of Economic studies 68, 235-260

Canton, E. (2002). Business cycles in a two-sector model of endogenous growth, Economic Theory 19, 477-492

Commission on Growth and Development (2008). The growth report: strategies for sustained growth and inclusive development (World Bank: Washington DC)

Dawid, H. (2006). Agent-based models of innovation and technological change, in (Tesfatsion, L., Judd, K.L., Eds) "Handbook of Computational Economics", Vol. 2, 1235-1272 (Amsterdam: Elsevier)

Diamond, P.A. (1982). Aggregate demand management in search equilibrium, Journal of Political Economy 90, 881-894

Dosi, G., Fagiolo, G., Roventini, A. (2010). Schumpeter meeting Keynes: a policy-friendly model of endogenous growth and business cycles, Journal of Economic Dynamics \& Contro 134, 1748-1767

Dosi, G., Nelson, R.R. (2010). Technical change and industrial dynamics as evolutionary processes, in (Hall, B.H., Rosenberg, N., Eds.) "Handbook of the Economics of Innovation", vol.1, 51-128 (Amsterdam: North-Holland) 
Dowrick, S. (1992). Technological catch up and diverging incomes: patterns of economic growth 1960-88, Economic Journal 102, 600-610

Eichengreen, B., Park, D., Shin, K. (2012).When fast economies slow down: international evidence and implications for China, Asian Economic Papers 11, 42-87

Eichengreen, B., Park, D., Shin, K. (2013).Growth slowdowns redux: new evidence on the middle-income trap, Working Paper No. 18673, National Bureau of Economic Research

Ethier, S. N. and Kurtz, T.G. (1986). Markov Processes: Characterization and Convergence, John Wiley and Sons

Evans, G.W., Honkapohja, S., Romer, P. (1998). Growth cycles, American Economic Review 88, 495-515

Furukawa, Y. (2007). Endogenous growth cycles, Journal of Economics 91, 69-96

Furukawa, Y. (2015). Leapfrogging cycles in international competition, Economic Theory 59, 401-433

Gill, I., Kharas, H. (2007).An east Asian renaissance: ideas for economic growth (World Bank: Washington, $\mathrm{DC})$

Grossman, G.M., Helpman, E. (1994). Endogenous innovation in the theory of growth, Journal of Economic Perspectives 8, 23-44

Hethcote, H.W. (2000). The mathematics of infectuous deseases, SIAM review 42, 599-653

Jones, C.I. (2005). Growth and ideas, in (Aghion, P., Durlauf, S., Eds.), "Handbook of Economic Growth", Vol. 1B, 1063-1111 (Amsterdam: Elsevier)

Kaas, L., Zink, S. (2007). Human capital and growth cycles, Economic Theory 31, 19-33

Kydland, F., Prescott, E. (1982) Time to build and aggregate fluctuations, Econometrica 50, 1345-1370

King, R,G., Plosser, C.I., Rebelo, S.T. (1988). Production, growth and business cycles: I. The basic neoclassical model, Journal of Monetary Economics 21, 195-232

King, R,G., Plosser, C.I., Rebelo, S.T. (1988). Production, growth and business cycles: II. New directions, Journal of Monetary Economics 21, 309-0341

Lahiri, A. (2001). Growth and equilibrium indeterminacy: the role of capital mobility, Economic Theory 17, $197-208$

La Torre, D., Liuzzi, D., Marsiglio, S. (2015). Pollution diffusion and abatement activities across space and over time, Mathematical Social Sciences 78, 48-63

Lewis, A.W. (1954). Economic development with unlimited supplies of labour, Manchester School 22, 139-191

Mathieu, P., Picco, P. (1998). Metastability and convergence to equilibrium for the random field Curie-Weiss model, Journal of Statistical Physics 91, 679-732

Marchese, C., Marsiglio, S., Privileggi, F., Ramello G.B. (2014). Endogenous recombinant growth through market production of knowledge and intellectual property rights, Dip. "Cognetti de Martiis" Working Paper No. 13/2014

Marsiglio, S. (2014). Reassessing Edgeworth's conjecture when population dynamics is stochastic, Journal of Macroeconomics 42, 130-140

Matsuyama, K. (1999). Growing through cycles, Econometrica 67, 335-347

Matsuyama, K. (2001). Growing through cycles in an infinitely lived agent economy, Journal of Economic Theory 100, 220-234

Mazzucato, M. (2013). The entrepreneurial state: debunking public vs. private sector myths (Anthem Press)

Mullingan, C.B., Sala-i-Martin, X. (1993). Transitional dynamics in two-sector models of endogenous growth, Quarterly Journal of Economics 108, 739-773

Nelson, R.R., Winter, S.G. (1982). An evolutionary theory of economic change (Cambridge: Belknap Press) 
Olivieri, R., Vares, M.E. (2005). Large deviations and metastability, Encyclopedia of Mathematics and its Applications, Vol.100 (Cambridge: Cambridge University Press)

Pritchett, L. (1997). Divergence, big time, Journal of Economic Perspectives 11, 3-17

Romer, P. (1986). Increasing returns and long-run growth, Journal of Political Economy 94, 1002-1037

Sachs, J.D., McArthur, J.W., Schimdt-Traub, G., Kruk, M., Bahadur, C., Faye, M., McCord, G. (2004). Ending Africa's poverty trap, Brookings Papers on Economic Activity 1, 117-240 (Washington: The Brookings Institution)

Smith, W.T. (2006). A closed form solution to the Ramsey model, Contributions to Macroeconomics 6, article 3

Skiba, A.K. (1978). Optimal growth with a convex-concave production function, Econometrica 46, 527-539

Walde, K. (2005). Endogenous growth cycles, International Economic Review 46, 867-894

Xie, D. (1994). Divergence in economic performance: transitional dynamics with multiple equilibria, Journal of Economic Theory 63, 97-112 